\title{
EVOLUTIONARY PROBLEMS IN NON-REFLEXIVE SPACES
}

\author{
MARTIN KRUŽÍK ${ }^{1,2}$ AND JOHANNES ZIMMER ${ }^{3}$
}

\begin{abstract}
Rate-independent problems are considered, where the stored energy density is a function of the gradient. The stored energy density may not be quasiconvex and is assumed to grow linearly. Moreover, arbitrary behaviour at infinity is allowed. In particular, the stored energy density is not required to coincide at infinity with a positively 1-homogeneous function. The existence of a rateindependent process is shown in the so-called energetic formulation.
\end{abstract}

Mathematics Subject Classification. 49J45, 35B05, 74G65.

Received June 27, 2007.

Published online October 21, 2008.

\section{INTRODUCTION}

The elastic-plastic behaviour of crystalline materials poses a challenge for mathematical analysis on the microscopic, mesoscopic and microscopic scale. Here, we study a rate-independent mesoscopic model with linear growth of the stored energy density at infinity. Such growth occurs, for instance, in the deformation theory of plasticity.

Before sketching the setting of the deformation theory of plasticity, we wish to describe the context of this study. A common and successful approach to the analysis of crystalline materials is via energy minimisation. This is manifest for elastic crystals, even for those with the potential of undergoing phase transitions [3]. For plastically deformed crystals, Ortiz and Repetto [19] list evidence that a variational approach is appropriate, and give a formulation in which dislocation structures can be understood as a nonconvex minimisation problem. The nature of this variational model is incremental, to reflect the inelastic and irreversible nature of plastic deformations [19]. We account for these phenomena with a phenomenological dissipation functional.

The applicability of variational methods has been broadened to include rate-independent evolution. A wealth of literature is available on this subject, and we refer the reader to the recent survey by Mielke [16]. Typically, these models are characterised by energy minimisation of a functional including macroscopic quantities such as the macroscopic deformation gradient as well as a dissipation functional.

Mielke and Roubíček [17] have combined this approach with the idea of describing micro-structured materials via Young measures. The application studied there is the rate-independent hysteretic evolution of shape-memory

\footnotetext{
Keywords and phrases. Concentrations, energetic solution, energies with linear growth, oscillations, relaxation.

1 Corresponding address: Institute of Information Theory and Automation, Academy of Sciences of the Czech Republic, Pod vodárenskou věží 4, 18208 Praha 8, Czech Republic.

2 Faculty of Civil Engineering, Czech Technical University, Thákurova 7, 16629 Praha 6, Czech Republic. kruzik@utia.cas.cz

3 Department of Mathematical Sciences, University of Bath, Bath BA2 7AY, UK. zimmer@maths.bath.ac.uk
} 
alloys under suitable external forcing. Here, we present an extension of this analysis to a setting where Young measures are not sufficient to describe the evolution.

It is known that Young measures are an appropriate tool to deal with oscillations, such as those arising in the description of microstructures. See Section 1.2 for a brief review. For problems with an energy that grows linearly in the argument at infinity, concentration effects may occur, which cannot be recorded with Young measures. DiPerna-Majda measures [7] can deal with such a situation; see Section 1.3 for a brief synopsis.

The use of DiPerna-Majda measures requires us to consider fine extensions of the Sobolev space $W^{1,1}(\Omega)$. An extension developed by Souček [22] turns out to be useful. Some relevant facts on this extension space, which might be of independent interest, are collected in Section 1.4.

The mathematical aim of this article is to establish a framework of rate-independent problems with energies of linear growth at infinity, and to prove the existence of the corresponding evolution of DiPerna-Majda measures. Energies with linear growth appear in a macroscopic (relaxed) model for single-crystal plasticity in the case of infinite latent hardening in the framework of the deformation theory of plasticity [4]. We recall that the deformation theory of plasticity is obtained when all material points follow certain optimal paths; this casts plasticity in a variational setting akin to elasticity [4]. For monotone proportional loading, this provides an appropriate description of plastic solids. We thus study the evolution of a material body under a (sufficiently small) load. For infinite latent hardening and linearised kinematics in the framework of the deformation theory of plasticity, it can be shown that the relaxation via convexification of a natural energy density is linear on the space of traceless symmetric matrices, and quadratic on the trace [4], Lemma 3.1. The linear growth is of particular interest, since it originates in a linear growth along the slip orbits of the unrelaxed functional. We thus restrict our attention to an energy of linear growth.

To the best of our knowledge, there is only one other study, by Dal Maso et al. of rate-independent evolutionary processes with linear or sublinear growth in the functional [5]. Though problems in plasticity are the motivation there as well as here, the models and consequently their analysis are rather different. Dal Maso and coworkers consider plasticity with softening, so that the sublinear growth is in the internal variable, rather than in the elastic-type energy, as is the case here. This difference is significant, as we consider a linearly growing energy, which depends on the deformation gradient; this differential constraint rules out the use of tools of convex analysis, which is the underlying thinking for many arguments of [5]. However, to prove existence, we have to employ a spatial regularisation, while the vanishing viscosity approach [5] allows Dal Maso et al. to prove the existence of a rate-independent evolution without regularisation.

Let us point out that we prove the existence of a rate-independent evolution directly via time discretisation and passage to the limit. In a recent interesting contribution, Dal Maso et al. develop a theory of timedependent DiPerna-Majda measures [6] and prove Helly's theorem for these measures. Though we do not make use of these results, it is possible that they provide an alternative route to the argument proving the existence of an evolutionary process.

This article is organised as follows. Some basic definitions are introduced in Section 1.1. Young measures respectively DiPerna-Majda measures are reviewed in Sections 1.2 and 1.3 respectively; Appendix 4 contains some relevant information regarding compactifications. The static problem is investigated in Section 2 , while the incremental problem is discussed in Section 3, and Section 4 describes the limit passage as the time discretisation goes to zero.

\subsection{Basic notation}

In this article, $\Omega \subset \mathbb{R}^{n}$ is always a bounded domain with smooth boundary. We denote the space of continuous functions $f: \Omega \rightarrow \mathbb{R}$ by $C(\Omega)$, while $C_{0}(\Omega)$ stands for the space of continuous functions $f: \Omega \rightarrow \mathbb{R}$ such that $\{x \in \Omega|| f(x) \mid \geq \epsilon\}$ is compact for every $\epsilon>0$.

Further, $W^{1,1}\left(\Omega ; \mathbb{R}^{m}\right)$ is as usual the space of measurable mappings which are integrable together with their first (distributional) derivatives; $W^{k, p}\left(\Omega ; \mathbb{R}^{m}\right)$ is defined analogously. We write w-lim for the weak limit. If $\Gamma_{D}$ is a part of the boundary $\partial \Omega$ with positive $n$-1-dimensional Hausdorff measure, $W_{u_{D}}^{1,1}\left(\Omega ; \mathbb{R}^{m}\right)$ stands for the 
set of functions $u \in W^{1,1}\left(\Omega ; \mathbb{R}^{m}\right)$ with $u=u_{D}$ on $\Gamma_{D}$. Weak convergence respectively weak-* convergence is expressed as $u_{k} \rightarrow u$ respectively $u_{k} \stackrel{\star}{\rightarrow} u$, while $u_{n} \rightarrow u$ denotes strong convergence.

\subsubsection{Measure theory}

Let us start with some basic definitions. If statements for a measure $\mu$ hold except for a set $N$ of measure zero, $\mu(N)=0$, this is abbreviated as $\mu$-almost all or $\mu$-a.e. If $X \subset \mathbb{R}^{n}$ is open and $\mu$ is the $n$-dimensional Lebesgue measure, then $\mu$ is omitted in the notation. For a measurable space $(X, \mathcal{M}, \mu)$, the usual Lebesgue space is denoted by $L^{1}(X, \mu)$. Again, we suppress $\mu$ from the notation if it is the Lebesgue measure.

We denote the (signed) Radon measures with finite mass on a locally compact Hausdorff space $X$ by $M(X)$; the cone of non-negative Radon measures with finite mass is denoted $M^{+}(X)$, and $\operatorname{Prob}(X)$ is the set of probability measures. The Jordan decomposition for signed measures $\mu=\mu^{+}-\mu^{-}$gives rise to the total variation $|\mu|$, which is the measure $|\mu|:=\mu^{+}+\mu^{-}$. Endowed with the total variation $\|\mu\|:=|\mu|(X)$ as a norm, $M(X)$ is a Banach space. By the Riesz representation theorem, $(M(X),\|\cdot\|)$ is isometrically isomorphic to the dual of $\left(C_{0}(X),\|\cdot\|_{\infty}\right)$ via the pairing

$$
\langle f, \mu\rangle:=\int_{\Omega} f(x) \mu(\mathrm{d} x) .
$$

The weak-* topology on $M(X)$ is defined by this duality. For $X \subset \mathbb{R}^{n}$, the singular part and the density of a Radon measure $\mu$ (given by the Lebesgue-Radon-Nikodym decomposition with respect to the Lebesgue measure) are denoted by $\mu_{s}$ and $\mu_{d}$, respectively.

The space of Radon measures with compact support on $\bar{\Omega}$ is denoted $(M(\bar{\Omega}),\|\cdot\|)$. We recall that the support of a Borel measure $\mu$ is the complement of the largest open set $N$ with $\mu(N)=0$.

We follow the convention of writing $C$ for a generic constant, whose value may change from line to line.

\subsection{Young measures}

We briefly recall the concept of Young measures. Young measures describe the limit of a sequence $\left\{u_{k}\right\}_{k \in \mathbb{N}}$ of functions $u_{k}: \Omega \rightarrow \mathbb{R}^{d}$ which converges weakly in $L^{p}\left(\Omega ; \mathbb{R}^{d}\right)$ for $1 \leq p<\infty$ or weakly* if $p=\infty$. The precise concept is as follows. A Young measure on a bounded domain $\Omega \subset \mathbb{R}^{n}$ is a weakly* measurable mapping

$$
\Omega \rightarrow \operatorname{Prob}\left(\mathbb{R}^{d}\right), \quad x \mapsto \nu_{x},
$$

with values in the probability measures. We recall that a mapping with values in the Radon measures is weakly* measurable if for any $f \in C_{0}\left(\mathbb{R}^{d}\right)$, the mapping

$$
\Omega \rightarrow \mathbb{R}, \quad x \mapsto\left\langle f, \nu_{x}\right\rangle:=\int_{\mathbb{R}^{d}} f(s) \nu_{x}(\mathrm{~d} s)
$$

is measurable in the usual sense. We denote the set of all Young measures by $\mathcal{Y}\left(\Omega ; \mathbb{R}^{d}\right)$. The analogous definition holds if $\mathbb{R}^{d}$ is replaced by a locally compact Hausdorff space $X$.

It is known that $\mathcal{Y}\left(\Omega ; \mathbb{R}^{d}\right)$ is a convex subset of $L_{\mathrm{w}}^{\infty}\left(\Omega ; M\left(\mathbb{R}^{d}\right)\right) \cong L^{1}\left(\Omega ; C_{0}\left(\mathbb{R}^{d}\right)\right)^{*}$, where $L_{\mathrm{w}}^{\infty}\left(\Omega ; M\left(\mathbb{R}^{d}\right)\right)$ is the space of weakly* measurable bounded functions. The parametrised Young measure theorem [23], Theorem 5 , states that for every sequence $\left\{u_{k}\right\}_{k \in \mathbb{N}}$ which is bounded in $L^{\infty}\left(\Omega ; \mathbb{R}^{d}\right)$, there exists a subsequence (denoted by the same indices for notational simplicity) and a Young measure $\nu=\left\{\nu_{x}\right\}_{x \in \Omega} \in \mathcal{Y}\left(\Omega ; \mathbb{R}^{d}\right)$ such that for every continuous function $f: \mathbb{R}^{d} \rightarrow \mathbb{R}$,

$$
f\left(u_{k}\right) \stackrel{\star}{\rightarrow} x \mapsto\left\langle f, \nu_{x}\right\rangle \text { weakly* in } L^{\infty}(\Omega),
$$

with

$$
\left\langle f, \nu_{x}\right\rangle:=\int_{\mathbb{R}^{d}} f(s) \nu_{x}(\mathrm{~d} s)
$$


being the expectation of $f$. Let $\mathcal{Y}^{\infty}\left(\Omega ; \mathbb{R}^{d}\right)$ denote set of all Young measures which are generated by taking all bounded sequences $\left\{u_{k}\right\}_{k \in \mathbb{N}}$ in $L^{\infty}\left(\Omega ; \mathbb{R}^{d}\right)$.

The above concept is applicable if $\left\{u_{k}\right\}_{k \in \mathbb{N}}$ is uniformly bounded in $L^{\infty}\left(\Omega ; \mathbb{R}^{d}\right)$. If in addition to the uniform bound in $L^{\infty}\left(\Omega ; \mathbb{R}^{d}\right), u_{k} \rightarrow u$ in $L^{p}\left(\Omega ; \mathbb{R}^{d}\right)$ with $1 \leq p<\infty$, then $u_{k} \rightarrow u$ if and only if the corresponding Young measure is a Dirac mass, $\nu_{x}=\delta_{u(x)}$. Non-Dirac Young measures thus record possible oscillations in the limit process.

The assumption that $\left\{u_{k}\right\}_{k \in \mathbb{N}}$ is bounded in $L^{\infty}\left(\Omega ; \mathbb{R}^{d}\right)$ can be relaxed to the assumption of such a bound in $L^{p}\left(\Omega ; \mathbb{R}^{d}\right)$ with $1<p<\infty$. The parametrised Young measure theorem is then valid under stronger growth conditions on the nonlinearity $f$. The precise formulation has been given by Schonbek [21], Theorem 2.2 (see also [2] for a general formulation of the parametrised Young measure theorem). Namely, for every sequence $\left\{u_{k}\right\}_{k \in \mathbb{N}}$ which is uniformly bounded in $L^{p}\left(\Omega ; \mathbb{R}^{d}\right)$ for some $p$ with $1<p<\infty$, there exists a subsequence, still indexed by $k$ for notational convenience, and a Young measure $\nu=\left\{\nu_{x}\right\}_{x \in \Omega} \in \mathcal{Y}\left(\Omega ; \mathbb{R}^{d}\right)$ such that for every $f \in C\left(\mathbb{R}^{d}\right)$ with

the following holds in $L^{1}\left(\Omega ; \mathbb{R}^{d}\right)$ :

$$
f(x)=o\left(|x|^{p}\right) \text { for }|x| \rightarrow \infty
$$

$$
f\left(u_{k}\right) \rightarrow x \mapsto\left\langle f, \nu_{x}\right\rangle .
$$

We say that $\left\{u_{k}\right\}_{k \in \mathbb{N}}$ generates $\nu$ if (1.4) holds; we denote the set of all Young measures obtained as limits of bounded sequences in $L^{p}\left(\Omega ; \mathbb{R}^{d}\right)$ by $\mathcal{Y}^{p}\left(\Omega ; \mathbb{R}^{d}\right)$.

\subsection{DiPerna-Majda measures}

In the situation under consideration, no bound in $L^{\infty}\left(\Omega ; \mathbb{R}^{d}\right)$ is available, and even the extension to bounds in $L^{p}\left(\Omega ; \mathbb{R}^{d}\right)$ for $1<p<\infty$ is not sufficient. Namely, the energy density $W$ will be a test function $f$ in the sense of (1.1). Obviously, a linearly growing energy density does not satisfy (1.3) even for $p=1$, and it is not hard to see that the bound (1.3) on the growth of the nonlinearity $f$ is sharp [21], Example 2.1. DiPerna-Majda measures are an extension of Young measures to describe concentration effects, which may occur due to the non-reflexivity of $L^{1}\left(\Omega ; \mathbb{R}^{d}\right)$. That is, let $f$ be a function $\mathbb{R}^{d} \rightarrow \mathbb{R}$ with $p$-growth at infinity. DiPerna-Majda measures then describe the limit of a sequence $\left\{f\left(u_{k}\right)\right\}_{k \in \mathbb{N}}$, where the functions $u_{k}: \Omega \rightarrow \mathbb{R}^{d}$ converge weakly in $L^{p}\left(\Omega ; \mathbb{R}^{d}\right)$ for $1 \leq p<\infty$, but are not uniformly bounded in $L^{\infty}\left(\Omega ; \mathbb{R}^{d}\right)$.

The definition of DiPerna-Majda measures involves a compactification; we refer to Appendix 4 for details and some intuition. There, we consider the motivation as to why we examine a completely regular subalgebra $\mathcal{F}$ of the space of bounded continuous functions $B C\left(\mathbb{R}^{d}\right)$; in the application, we will set $d:=m n$.

We consider compactifications $\beta_{\mathcal{F}} \mathbb{R}^{d}$ by a sphere or finer. That is, $\mathcal{F}$ contains all functions $\tilde{f}$ for which the radial $\operatorname{limit}_{\lim } \lim _{r \rightarrow \infty} \tilde{f}(r s)$ exists for arbitrary $s \in \mathbb{R}^{d}$. We note that $\mathcal{F}$ also may contain functions $\tilde{f}$ which have no well-defined radial limits. To deal with functions $f$ with linear growth at infinity in a convenient manner, we set $\tilde{f}(s):=\frac{f(s)}{1+|s|}$, with $\tilde{f} \in \mathcal{F}$.

The motivation for the construction of DiPerna-Majda measures can be described as follows. We are given a sequence $\left\{u_{k}\right\}_{k \in \mathbb{N}}$, uniformly bounded in $L^{p}\left(\Omega, \mathbb{R}^{d}\right)$. For the application discussed below, it suffices to consider the case $p=1$. The goal is to describe the weak limit

$$
\lim _{k \rightarrow \infty} \int_{\Omega} \phi(x) f\left(u_{k}(x)\right) \mathrm{d} x,
$$

with $\phi \in C_{0}(\Omega)$ and $f(s):=\tilde{f}(s)(1+|s|)$, where $\tilde{f} \in B C\left(\mathbb{R}^{d}\right)$. A canonical norm for $f$ of this form is $|f|_{\infty}:=\max _{s \in \mathbb{R}^{d}} \tilde{f}(s)=|\tilde{f}|_{\infty}$.

DiPerna and Majda have shown the following results for open domains $\Omega$ and test functions $\phi \in C_{0}(\Omega)$. We state the results for $\bar{\Omega}$ and test functions $\phi \in C(\bar{\Omega})$. The proofs remain the same, except that the isomorphism between the dual space of $\left(C_{0}(\Omega),\|\cdot\|\right)$ and the space $(M(\Omega),\|\cdot\|)$ of Radon measures with finite mass has 
to be replaced by the isomorphism of $(C(\bar{\Omega}),\|\cdot\|)$ and the space of Radon measures with compact support $(M(\bar{\Omega}),\|\cdot\|)$.

For a bounded sequence $\left\{u_{k}\right\}_{k \in \mathbb{N}}$ in $L^{1}\left(\bar{\Omega} ; \mathbb{R}^{d}\right)$, there exists a non-negative Radon measure $\sigma \in M^{+}(\bar{\Omega})$ such that

$$
\left(1+\left|u_{k}(x)\right|\right) \mathrm{d} x \stackrel{\star}{\rightarrow} \sigma \text { in } M(\bar{\Omega}) ;
$$

see [7], Theorem 4.1. Furthermore, for a separable completely regular subalgebra $\mathcal{F}$ of $B C\left(\mathbb{R}^{d}\right)$, there exist a $\sigma$-measurable map $\hat{\nu}: \Omega \rightarrow \operatorname{Prob}\left(\beta_{\mathcal{F}} \mathbb{R}^{d}\right), x \mapsto \hat{\nu}_{x}$, and a subsequence of $\left\{u_{k}\right\}_{k \in \mathbb{N}}$ (not relabeled) such that for every $\tilde{f} \in \mathcal{F}$

$$
\lim _{k \rightarrow \infty} \int_{\bar{\Omega}} \phi(x) f\left(u_{k}(x)\right) \mathrm{d} x=\int_{\bar{\Omega}} \phi(x) \int_{\beta_{\mathcal{F}} \mathbb{R}^{d}} \tilde{f}(s) \hat{\nu}_{x}(\mathrm{~d} s) \sigma(\mathrm{d} x)
$$

holds for every $\phi \in C(\bar{\Omega})[7]$, Theorem 4.3. We say that $\left\{u_{k}\right\} \in \mathbb{N}$ generates the pair $(\sigma, \hat{\nu})$ if equation (1.6) holds. A pair $(\sigma, \hat{\nu}) \in M^{+}(\bar{\Omega}) \times L_{\mathrm{w}}^{\infty}\left(\bar{\Omega}, \sigma ; \operatorname{Prob}\left(\beta_{\mathcal{F}} \mathbb{R}^{d}\right)\right)$ attainable by sequences in $L^{1}\left(\Omega ; \mathbb{R}^{d}\right)$ is called a DiPerna-Majda measure. The set of all DiPerna-Majda measures is denoted $\mathcal{D} \mathcal{M}_{\mathcal{F}}\left(\Omega ; \mathbb{R}^{d}\right)$.

The explicit description of the elements of $\mathcal{D} \mathcal{M}_{\mathcal{F}}\left(\Omega ; \mathbb{R}^{d}\right)$ for unconstrained sequences is given in [14], Theorem 2. The characterisation of DiPerna-Majda measures generated by gradients of Sobolev maps in $W^{1, p}\left(\Omega ; \mathbb{R}^{m}\right)$ for $p>1$ can be found in [13].

It is sometimes convenient to consider an alternative representation of DiPerna-Majda measures. Specifically, in analogy to the proof of Theorem 4.1 in [7], we will consider measures in $M\left(\bar{\Omega} \times \beta_{\mathcal{F}} \mathbb{R}^{d}\right)$. We say that $\left\{u_{k}\right\}_{k \in \mathbb{N}} \subset L^{1}\left(\bar{\Omega} ; \mathbb{R}^{d}\right)$ generates the measure $\eta \in M\left(\bar{\Omega} \times \beta_{\mathcal{F}} \mathbb{R}^{d}\right)$ if, for every $\tilde{h} \in C\left(\bar{\Omega} \times \beta_{\mathcal{F}} \mathbb{R}^{d}\right)$,

$$
\lim _{k \rightarrow \infty} \int_{\bar{\Omega}} \tilde{h}\left(x, u_{k}(x)\right)\left(1+\left|u_{k}(x)\right|\right) \mathrm{d} x=\int_{\bar{\Omega} \times \beta_{\mathcal{F}} \mathbb{R}^{d}} \tilde{h}(x, s) \eta(\mathrm{d} s \mathrm{~d} x)
$$

holds. The set of all measures generated in this way will be denoted $D M_{\mathcal{F}}\left(\Omega ; \mathbb{R}^{d}\right)$. Since $\phi(x) \tilde{f}(y)$ with $\phi \in C(\bar{\Omega})$ and $\tilde{f} \in B C\left(\beta_{\mathcal{F}} \mathbb{R}^{d}\right)$ is dense in $C\left(\bar{\Omega} \times \beta_{\mathcal{F}} \mathbb{R}^{d}\right)$, one can say that $\eta \cong(\sigma, \hat{\nu})$ for $\eta \in D M_{\mathcal{F}}\left(\Omega ; \mathbb{R}^{d}\right)$ and $(\sigma, \hat{\nu}) \in \mathcal{D} \mathcal{M}_{\mathcal{F}}\left(\Omega ; \mathbb{R}^{d}\right)$ if

$$
\langle\tilde{h}, \eta\rangle:=\int_{\bar{\Omega} \times \beta_{\mathcal{F}} \mathbb{R}^{d}} \tilde{h}(x, s) \eta(\mathrm{d} x \mathrm{~d} s)=\int_{\bar{\Omega}} \int_{\beta_{\mathcal{F}} \mathbb{R}^{d}} \tilde{h}(x, s) \hat{\nu}_{x}(\mathrm{~d} s) \sigma(\mathrm{d} x)
$$

for any $\tilde{h} \in C\left(\bar{\Omega} \times \beta_{\mathcal{F}} \mathbb{R}^{d}\right)$. Consequently, the elements of $D M_{\mathcal{F}}\left(\Omega ; \mathbb{R}^{d}\right)$ will be addressed as DiPerna-Majda measures as well.

It is known [20], Chapter 3 , that $D M_{\mathcal{F}}\left(\Omega ; \mathbb{R}^{d}\right)$ is a closed, convex, non-compact but locally compact and locally sequentially compact subset of the locally convex space $M\left(\bar{\Omega} \times \beta_{\mathcal{F}} \mathbb{R}^{d}\right)$ considered in its weak ${ }^{*}$ topology.

We denote by $\mathcal{G} \mathcal{D} \mathcal{M}_{\mathcal{F}}\left(\Omega ; \mathbb{R}^{m \times n}\right)$ the subset of $\mathcal{D} \mathcal{M}_{\mathcal{F}}\left(\Omega ; \mathbb{R}^{m \times n}\right)$ of those measures which are generated by gradients of mappings in $W^{1,1}\left(\Omega ; \mathbb{R}^{m}\right)$. Expressed differently, $(\sigma, \hat{\nu}) \in \mathcal{G D} \mathcal{M}_{\mathcal{F}}\left(\Omega ; \mathbb{R}^{m \times n}\right)$ if there is $\left\{u_{k}\right\}_{k \in \mathbb{N}} \subset$ $W^{1,1}\left(\Omega ; \mathbb{R}^{m}\right)$ such that for all $\phi \in C(\bar{\Omega})$ and all $\tilde{f} \in \mathcal{F}$

$$
\lim _{k \rightarrow \infty} \int_{\bar{\Omega}} \phi(x) f\left(\nabla u_{k}(x)\right) \mathrm{d} x=\int_{\bar{\Omega}} \int_{\beta_{\mathcal{F}} \mathbb{R}^{m \times n}} \phi(x) \tilde{f}(s) \hat{\nu}_{x}(\mathrm{~d} s) \sigma(\mathrm{d} x) .
$$

Similarly we write $\eta \in G D M_{\mathcal{F}}\left(\Omega ; \mathbb{R}^{m \times n}\right)$ if $\eta \in D M_{\mathcal{F}}\left(\Omega ; \mathbb{R}^{m \times n}\right)$ is generated by gradients. Finally, $\mathcal{G D} \mathcal{M}_{\mathcal{F}}^{u_{D}}\left(\Omega ; \mathbb{R}^{m \times n}\right)$ denotes elements $(\sigma, \hat{\nu}) \in \mathcal{G} \mathcal{D} \mathcal{M}_{\mathcal{F}}\left(\Omega ; \mathbb{R}^{m \times n}\right)$ with the property that $(\sigma, \hat{\nu})$ is generated by $\left\{u_{k}\right\}_{k \in \mathbb{N}} \subset W_{u_{D}}^{1,1}\left(\Omega ; \mathbb{R}^{m}\right)$, with $u_{D} \in W^{1,1}\left(\Omega ; \mathbb{R}^{m}\right)$.

\subsection{Fine extensions of $W^{\mathbf{1 , 1}}\left(\Omega ; \mathbb{R}^{\mathbf{m}}\right)$}

As $W^{1,1}\left(\Omega ; \mathbb{R}^{m}\right)$ is not a reflexive space, we shall work with a weak ${ }^{*}$ compact extension. We mention two extensions. The first one is the space of functions with bounded variation $B V\left(\Omega ; \mathbb{R}^{m}\right)($ see, e.g., $[1,10])$. 
The space $B V\left(\Omega ; \mathbb{R}^{m}\right)$ is the linear subspace of $L^{1}\left(\Omega ; \mathbb{R}^{m}\right)$ containing maps with bounded variation in $\Omega$. That is, $u \in B V\left(\Omega ; \mathbb{R}^{m}\right)$ if

$$
\|u\|_{B V\left(\Omega ; \mathbb{R}^{m}\right)}:=\|u\|_{L^{1}\left(\Omega ; \mathbb{R}^{m}\right)}+\|D u\|_{M\left(\Omega ; \mathbb{R}^{m \times n}\right)}<\infty
$$

where, with $\Phi:=\left\{\varphi \in C_{0}^{1}\left(\Omega ; \mathbb{R}^{m \times n}\right)|| \varphi \mid \leq 1\right\}$,

$$
\|D u\|_{M\left(\Omega ; \mathbb{R}^{m \times n)}\right.}:=\sup _{\varphi \in \Phi} \int_{\Omega} u \cdot \operatorname{div} \varphi \mathrm{d} x
$$

It is easy to see that $\|\cdot\|_{B V\left(\Omega ; \mathbb{R}^{m}\right)}$ defines the norm on $B V\left(\Omega ; \mathbb{R}^{m}\right)$.

We use weak*-convergence in $B V\left(\Omega ; \mathbb{R}^{m}\right)$. A sequence $\left\{u_{k}\right\}_{k \in \mathbb{N}} \subset B V\left(\Omega ; \mathbb{R}^{m}\right)$ is said to converge weakly* in $B V\left(\Omega ; \mathbb{R}^{m}\right)$ to $u$ if $u_{k} \rightarrow u$ in $L^{1}\left(\Omega ; \mathbb{R}^{m}\right)$ and $D u_{k} \rightarrow D u$ weakly* in the sense of measures as $k \rightarrow \infty$, i.e.,

$$
\lim _{k \rightarrow \infty} \int_{\Omega} \phi(x) D u_{k}(\mathrm{~d} x)=\int_{\Omega} \phi(x) D u(\mathrm{~d} x)
$$

for every $\phi \in C_{0}(\Omega)[1]$, Definition 3.11.

Finally, if $\partial \Omega$ is Lipschitz then there is a bounded linear mapping $T: B V\left(\Omega ; \mathbb{R}^{m}\right) \rightarrow L^{1}\left(\partial \Omega ; \mathbb{R}^{m}\right)$ such that (here and in the following, $\nu$ is the unit outer normal to $\partial \Omega \subset \mathbb{R}^{n}$ )

$$
\int_{\partial \Omega}(\varphi \cdot \nu) T u^{j}(\mathrm{~d} A)=\int_{\Omega} u^{j}(x) \operatorname{div} \varphi(x) \mathrm{d} x+\int_{\Omega} \varphi \cdot\left[D u^{j}\right](\mathrm{d} x)
$$

for all $\varphi \in C^{1}\left(\mathbb{R}^{n} ; \mathbb{R}^{n}\right)$ and all $1 \leq j \leq m[10]$, Theorem 5.3.1. The measure $\bar{T} u=\left(\bar{T} u^{1}, \ldots, \bar{T} u^{m}\right)$ is called the trace of $u \in B V\left(\Omega ; \mathbb{R}^{m}\right)$.

The second extension was developed by Souček $[22]$; we denote it by $W^{1, \mu}\left(\bar{\Omega} ; \mathbb{R}^{m}\right)$. This extension consists of functions in $L^{1}\left(\Omega ; \mathbb{R}^{m}\right)$ whose gradient is a measure on $\bar{\Omega}$. More precisely,

$$
\begin{gathered}
W^{1, \mu}\left(\Omega ; \mathbb{R}^{m}\right)=\left\{(u, \bar{D} u) \in L^{1}\left(\Omega ; \mathbb{R}^{m}\right) \times M(\bar{\Omega}) ; \text { there exists }\left\{u_{k}\right\}_{k \in \mathbb{N}} \subset W^{1,1}\left(\Omega ; \mathbb{R}^{m}\right)\right. \text { such that } \\
\left.u_{k} \rightarrow u \text { in } L^{1}\left(\Omega ; \mathbb{R}^{m}\right) \text { and } \nabla u_{k} \rightarrow \bar{D} u \text { weakly* in } M\left(\bar{\Omega} ; \mathbb{R}^{m \times n}\right)\right\} .
\end{gathered}
$$

It is known [22] that $W^{1, \mu}\left(\Omega ; \mathbb{R}^{m}\right)$ is a Banach space if it is normed by

$$
\|(u, \bar{D} u)\|_{W^{1, \mu}\left(\Omega ; \mathbb{R}^{m}\right)}=\|u\|_{L^{1}\left(\Omega ; \mathbb{R}^{m}\right)}+\|\bar{D} u\|_{M\left(\bar{\Omega} ; \mathbb{R}^{m \times n}\right)} .
$$

The weak* convergence in $W^{1, \mu}\left(\Omega ; \mathbb{R}^{m}\right)$ is defined analogously to $B V\left(\Omega ; \mathbb{R}^{m}\right)$. The precise formulation is that (1.9) has to hold with $\bar{D} u$ instead of $D u$ for any $\phi \in C(\bar{\Omega})$. Moreover, as shown in [22], Theorem 1 (iii), if $(u, D u) \in W^{1, \mu}\left(\Omega ; \mathbb{R}^{m}\right)$, then there is a unique measure $\bar{T}(u, \bar{D} u) \in M\left(\partial \Omega ; \mathbb{R}^{m}\right)$ such that

$$
\int_{\partial \Omega}(\varphi \cdot \nu)\left[\bar{T}\left(u^{j}, \bar{D} u^{j}\right)\right](\mathrm{d} A)=\int_{\Omega} u^{j}(x) \operatorname{div} \varphi(x) \mathrm{d} x+\int_{\bar{\Omega}} \varphi \cdot\left[\bar{D} u^{j}\right](\mathrm{d} x)
$$

for all $\varphi \in C^{1}\left(\bar{\Omega} ; \mathbb{R}^{n}\right)$ and all $1 \leq j \leq m$. The measure $\bar{T}(u, \bar{D} u)=\left(\bar{T}\left(u^{1}, \bar{D} u^{1}\right), \ldots, \bar{T}\left(u^{m}, \bar{D} u^{m}\right)\right)$ is called the trace of $(u, \bar{D} u)$. Here, the measure $\bar{D} u^{j}$ denotes the $j$ th row of the matrix-valued measure $\bar{D} u$. The operator $W^{1, \mu}\left(\Omega ; \mathbb{R}^{m}\right) \rightarrow M\left(\partial \Omega ; \mathbb{R}^{m}\right)$ given by $(u, D u) \mapsto \bar{T} u$ is (weak ${ }^{*}$, weak $\left.{ }^{*}\right)$ continuous [22], Theorem 2 (ii). Finally, balls in $W^{1, \mu}\left(\Omega ; \mathbb{R}^{m}\right)$ are weakly* compact [22], Theorem 6 .

It is easy to see that $W^{1, \mu}\left(\Omega ; \mathbb{R}^{m}\right)$ is a finer extension of $W^{1,1}\left(\Omega ; \mathbb{R}^{m}\right)$ than $B V\left(\Omega ; \mathbb{R}^{m}\right)$. Namely, Du is the restriction of $\bar{D} u$ on $\Omega$. Hence, one can define a projection $P: W^{1, \mu}\left(\Omega ; \mathbb{R}^{m}\right) \rightarrow B V\left(\Omega ; \mathbb{R}^{m}\right)$ by $P(u, \bar{D} u)=$ $(u, D u)$, with $D u=\left.\bar{D} u\right|_{\Omega}$. 
A comparison of $(1.11)$ with $(1.10)$ reveals that for all $\varphi \in C^{1}\left(\mathbb{R}^{n} ; \mathbb{R}^{n}\right)$

$$
\int_{\partial \Omega}(\varphi \cdot \nu)\left(\left[\bar{T}\left(u^{j}, \bar{D} u^{j}\right)\right](\mathrm{d} A)-T u^{j} \mathrm{~d} A\right)=\int_{\partial \Omega} \varphi \cdot\left[\bar{D} u^{j}\right](\mathrm{d} x)
$$

i.e., if $\bar{D} u^{i}$ does not concentrate on $\partial \Omega$, then the BV notion of trace coincides with the one in the Souček space $W^{1, \mu}\left(\Omega ; \mathbb{R}^{m}\right)$.

Consider $(u, \bar{D} u) \in W^{1, \mu}\left(\Omega ; \mathbb{R}^{m}\right)$ and define a measure $\bar{D} y$ on $\bar{\Omega}$ as follows: $\bar{D} y:=\bar{D} u$ on $\partial \Omega$ and $\bar{D} y=0$ on $\Omega$. Finally, define $\bar{D} z:=\bar{D} u-\bar{D} y$. Then $(u, \bar{D} z),(0, \bar{D} y) \in W^{1, \mu}\left(\Omega ; \mathbb{R}^{m}\right)[22]$, Theorem 8. Hence,

$$
(u, \bar{D} u)=(u, \bar{D} z)+(0, \bar{D} y)
$$

As $\bar{D} z$ does not concentrate on $\partial \Omega$, it follows from (1.12) that

$$
\bar{T}(u, \bar{D} u)=T u+\bar{T}(0, \bar{D} y) .
$$

We have the following Poincaré-type inequality.

Lemma 1.1. Let $\Omega \subset \mathbb{R}^{n}$ a be bounded domain, with $\partial \Omega$ belonging to class $C^{1}$. Let $\Gamma_{D} \subset \partial \Omega$ be open and of positive $n$-1-dimensional Lebesgue measure; suppose further that $z \in M\left(\Gamma_{D} ; \mathbb{R}^{m}\right)$. Then there is $C>0$ such that the estimate

$$
\|u\|_{L^{1}\left(\Omega ; \mathbb{R}^{m}\right)} \leq C\left(\|\bar{D} u\|_{M\left(\bar{\Omega} ; \mathbb{R}^{m \times n}\right)}+\|z\|_{M\left(\Gamma_{D} ; \mathbb{R}^{m}\right)}\right)
$$

holds for all $(u, \bar{D} u) \in W^{1, \mu}\left(\Omega ; \mathbb{R}^{m}\right)$ with $\bar{T}(u, \bar{D} u)=z$ on $\Gamma_{D}$.

Proof. Suppose that (1.13) does not hold. This means that for all $k \in \mathbb{N}$ there is $\left(u_{k}, \bar{D} u_{k}\right) \in W^{1, \mu}\left(\Omega ; \mathbb{R}^{m}\right)$ with $\bar{T}\left(u_{k}, \bar{D} u_{k}\right)=z$ on $\Gamma_{D}$ such that

$$
\left\|u_{k}\right\|_{L^{1}\left(\Omega ; \mathbb{R}^{m}\right)}>k\left(\left\|\bar{D} u_{k}\right\|_{M\left(\bar{\Omega} ; \mathbb{R}^{m \times n}\right)}+\|z\|_{M\left(\Gamma_{D} ; \mathbb{R}^{m}\right)}\right) .
$$

Let us put $v_{k}:=\frac{u_{k}}{\left\|u_{k}\right\|_{L^{1}\left(\Omega ; \mathbb{R}^{m}\right)}}$ and $\bar{D} v_{k}:=\frac{\bar{D} u_{k}}{\left\|u_{k}\right\|_{L^{1}\left(\Omega ; \mathbb{R}^{m}\right)}}$. Then the last inequality implies

$$
1>k\left(\left\|\bar{D} v_{k}\right\|_{M\left(\bar{\Omega} ; \mathbb{R}^{m \times n}\right)}+\left\|u_{k}\right\|_{L^{1}\left(\Omega ; \mathbb{R}^{m}\right)}^{-1}\|z\|_{M\left(\Gamma_{D} ; \mathbb{R}^{m}\right)}\right)
$$

In particular, we have $\left\|v_{k}\right\|_{L^{1}\left(\Omega ; \mathbb{R}^{m}\right)}=1$ and $\left\|\bar{D} v_{k}\right\|_{M(\bar{\Omega})} \leq \frac{1}{k}$. Consequently, for all $k \in \mathbb{N}$, $\left\|\left(v_{k}, \bar{D} v_{k}\right)\right\|_{W^{1, \mu}\left(\Omega ; \mathbb{R}^{m}\right)} \leq 2$. The weak* compactness of balls in $W^{1, \mu}\left(\Omega ; \mathbb{R}^{m}\right)$ implies that there is $(v, \bar{D} v) \in$ $W^{1, \mu}\left(\Omega ; \mathbb{R}^{m}\right)$ such that for a subsequence (not relabeled) $w^{*}-\lim _{k \rightarrow \infty}\left(v_{k}, \bar{D} v_{k}\right)=(v, \bar{D} v)$. Moreover, $\|v\|_{L^{1}\left(\Omega ; \mathbb{R}^{m}\right)}=1$ and $\bar{D} v=0$. Finally, the weak ${ }^{*}$ continuity of the trace operator and the fact that $\left\|u_{k}\right\|_{L^{1}\left(\Omega ; \mathbb{R}^{m}\right)} \rightarrow$ $\infty$ imply that $\bar{T}(v, \bar{D} v)=0$ on $\Gamma_{D}$. As $\bar{D} v=0$, we have that $v$ is constant and furthermore $v \in W^{1,1}\left(\Omega ; \mathbb{R}^{m}\right)$. On the other hand, $\bar{T}(v, \bar{D} v)=0$, i.e., $v=0$. This, however, contradicts the fact that $\|v\|_{L^{1}\left(\Omega ; \mathbb{R}^{m}\right)}=1$.

Remark 1.2. For the validity of Lemma 1.1, it is important that $\Gamma_{D}$ is open in $\partial \Omega$. Namely, the weak* continuity of $\bar{T}$ implies that $\bar{T}\left(v_{k}, \bar{D} v_{k}\right) \rightarrow \bar{T}(v, \bar{D} v)$ weakly* in $M\left(\partial \Omega ; \mathbb{R}^{m}\right)$. Clearly, this does not necessarily imply that $\bar{T}\left(v_{k}, \bar{D} v_{k}\right) \rightarrow \bar{T}(v, \bar{D} v)$ weakly* in $M\left(\Gamma_{D} ; \mathbb{R}^{m}\right)$ for $\Gamma_{D}$ not open in $\partial \Omega$.

Finally, we would like to mentioned that $W^{1, \mu}\left(\Omega ; \mathbb{R}^{m}\right)$ is compactly embedded into $L^{q}\left(\Omega ; \mathbb{R}^{m}\right)$ for all $1 \leq$ $q<\frac{n}{n-1}$ [22], Theorem 5 . 


\subsection{Existence of a rate-independent process}

The aim of this article is to prove the existence of a rate-independent process for a suitable relaxation of a problem involving a stored energy that grows linearly in the argument of the gradient. We wish to state the result before embarking on the lengthy proof. To do so, we need to state some definitions.

Let $Q$ be the set of admissible configurations; this is defined precisely in (3.5). Then we define the Gibbs stored energy as

$$
\Gamma(t, q):=\int_{\bar{\Omega} \times \beta_{\mathcal{F}} \mathbb{R}^{m \times n}} \tilde{W}(x, s) \eta(\mathrm{d} s \mathrm{~d} x)+\varrho\|\lambda(x)\|_{W^{1,2}\left(\Omega ; \mathbb{R}^{L}\right)}-\int_{\Omega} f(x, t) \cdot u(x) \mathrm{d} x
$$

$(\Gamma(t, q)$ is set to be $\infty$ for non-admissible states as well as some states with a lack of regularity, see Eq. (3.10)). Here, $\tilde{W}(x, s):=\frac{W(x, s)}{1+|s|}$, and $W$ itself satisfies the linear bounds $(2.1)$ at infinity. Furthermore, $\varrho\|\lambda(x)\|_{W^{1,2}\left(\Omega ; \mathbb{R}^{L}\right)}$ is a regularisation discussed in Section 3.

We restrict ourselves to an external loading that depends continuously on time, $f \in W^{1,1}\left([0, T] ; L^{p}\left(\Omega ; \mathbb{R}^{m}\right)\right)$, with $p>n$. For an admissible state $q$, we shall write

$$
F(t, q):=\int_{\Omega} f(x, t) \cdot u(x) \mathrm{d} x
$$

for the contribution of the external load to the Gibbs energy ( $u$ is one component of $q$ ).

We follow the conventional procedure of introducing a phenomenological dissipation distance $D$; the assumptions on $D$ are listed in Definition 3.1 (see also Eq. (3.6)); furthermore, (4.1) imposes one more condition on the dissipation. Finally, for a process $q:[0, T] \rightarrow Q$ and a given time interval $\left[t_{1}, t_{2}\right] \subset[0, T]$, the temporal dissipation is given by

$$
\operatorname{Diss}\left(q,\left[t_{1}, t_{2}\right]\right):=\sup _{L \in \mathbb{N}}\left\{\sum_{l=1}^{L} D\left(\eta\left(\tau_{l-1}\right), \eta\left(\tau_{l}\right)\right) \mid t_{1}=\tau_{0}<\tau_{2}<\cdots<\tau_{L}=t_{2}\right\} .
$$

The notion of a solutions to energetic models can be stated as follows (see $[17,18]$ ).

Definition 1.3. Given $q_{0} \in Q$ we say that the process $q:[0, T] \rightarrow Q$ will be a solution if the following conditions hold:

(1) $(u, \bar{D} u) \in L^{\infty}\left(0, T ; W^{1, \mu}\left(\Omega ; \mathbb{R}^{m}\right)\right)$.

(2) $\lambda \in B V\left(0, T ; L^{1}\left(\Omega ; \mathbb{R}^{L}\right)\right)$, where equation (3.3) gives the precise definition of $\lambda$ (we initially work with $\lambda \in B V\left(0, T ; M\left(\bar{\Omega} ; \mathbb{R}^{L}\right)\right)$; see the discussion before Def. 3.3 for more details).

(3) Global stability: For every $t \in[0, T]$, we have

$$
\Gamma(t, q(t)) \leq \Gamma(t, \tilde{q})+D(q(t), \tilde{q}) \text { for every } \tilde{q} \in Q
$$

(4) Energy inequality: For every $0 \leq t_{1} \leq t_{2} \leq T$, we have

$$
\Gamma\left(t_{1}, q\left(t_{1}\right)\right)+\operatorname{Diss}\left(q,\left[t_{1}, t_{2}\right]\right) \leq \Gamma\left(t_{2}, q\left(t_{2}\right)\right)-\int_{t_{1}}^{t_{2}} \dot{F}(t, q(t)) \mathrm{d} t .
$$

(5) Initial condition: $q(0)=q_{0}$.

The main result of this article can now be stated as follows.

Theorem 1.4. Under the assumptions listed in this subsection, for sufficiently small forcing $f$ (see Prop. 4.2 for the precise formulation), there exists a rate-independent process which is a solution in the sense of Definition 1.3. 
The proof of this theorem is broken into a string of arguments, investigating first the static situation in Section 2, then a time-discretisation in Section 3, and finally the passage to the limit of vanishing time discretisation in Section 4.

\section{Static Problem}

We now focus on a static problem with a linearly growing energy in the variational formulation. The setting is as follows. We are given a continuous function $W: \bar{\Omega} \times \mathbb{R}^{n \times m} \rightarrow \mathbb{R}$ such that there exist constants $\beta \geq \alpha>0$ with

$$
\alpha|s|-\beta \leq W(x, s) \leq \beta(1+|s|) \text { for every } x \in \bar{\Omega} .
$$

Further, the analysis includes a forcing term

$$
f \in L^{p}\left(\Omega ; \mathbb{R}^{m}\right)
$$

with $p>n$; precise assumptions on the smallness of this forcing are stated later in this section.

Then, the variational problem consists of finding

$$
\text { Minimise } I(u):=\int_{\Omega} W(x, \nabla u(x)) \mathrm{d} x-\int_{\Omega} f(x) \cdot u(x) \mathrm{d} x \text { among } u \in W_{u_{D}}^{1,1}\left(\Omega ; \mathbb{R}^{m}\right) \text {. }
$$

We recall that $W_{u_{D}}^{1,1}\left(\Omega ; \mathbb{R}^{m}\right)$ is the set of functions $u \in W^{1,1}\left(\Omega ; \mathbb{R}^{m}\right)$ with $u=u_{D}$ on $\Gamma_{D}$ in the sense of traces.

We do not assume that $W$ is quasiconvex in $s$ and thus have to resort to a relaxed formulation of (2.3) in the space of DiPerna-Majda measures. Yet, we point out that even for convex $W(x, \cdot)$, there may be no solution. This is demonstrated in the following example.

Example 2.1. Take $m=n=1, \Omega=(0,1), f=0, W(x, s)=\left(x-\frac{1}{2}\right)^{2}|s|, u(0)=0$ and $u(1)=1$. Then $\inf I=0$. Indeed, an infimising sequence may look as follows

$$
u_{k}(x)= \begin{cases}0 & \text { if } x \in\left(0, \frac{1}{2}-\frac{1}{2 k}\right] \\ k x-\frac{k}{2}+\frac{1}{2} & \text { if } x \in\left(\frac{1}{2}-\frac{1}{2 k}, \frac{1}{2}+\frac{1}{2 k}\right) \\ 1 & \text { if } x \in\left(\frac{1}{2}+\frac{1}{2 k}, 1\right]\end{cases}
$$

It is immediate that $\lim _{k \rightarrow \infty} I\left(u_{k}\right)=0$, but $\min I$ does not exist.

Before stating the relaxed version of the static problem (2.3), we have to collect an auxiliary statement that permits us to recover information regarding a function $u$ whose measure derivative, $D u$, is the first moment of a gradient DiPerna-Majda measure.

Lemma 2.2. Let $\left\{u_{k}\right\}_{k \in \mathbb{N}} \subset W_{u_{D}}^{1,1}\left(\Omega ; \mathbb{R}^{m}\right)$ be such that $\left\{\nabla u_{k}\right\}_{k \in \mathbb{N}}$ generates $(\sigma, \hat{\nu}) \in \mathcal{G D} \mathcal{M}_{\mathcal{F}}^{u_{D}}\left(\Omega ; \mathbb{R}^{m \times n}\right)$. Then there is $(u, \bar{D} u) \in W^{1, \mu}\left(\Omega ; \mathbb{R}^{m}\right)$ and a subsequence (not relabeled) such that $u_{k} \rightarrow u$ in $L^{1}\left(\Omega ; \mathbb{R}^{m}\right)$. Furthermore, $(u, \bar{D} u)$ satisfies $\bar{T}(u, \bar{D} u)=u_{D}$ on $\Gamma_{D}$, and $u$ is a unique solution to

$$
\int_{\bar{\Omega}} \phi(x) \bar{D} u \mathrm{~d} x=\int_{\bar{\Omega}} \phi(x) \int_{\beta_{\mathcal{F}} \mathbb{R}^{m \times n}} \frac{s}{1+|s|} \hat{\nu}_{x}(\mathrm{~d} s) \sigma(\mathrm{d} x)
$$

for every $\phi \in C(\bar{\Omega})$, i.e., $\bar{D} u=\int_{\beta_{\mathcal{F}} \mathbb{R}^{m \times n}} \frac{s}{1+|s|} \hat{\nu}_{x}(\mathrm{~d} s)$ in the sense of measures on $\bar{\Omega}$.

Proof. As $\left\{\nabla u_{k}\right\}_{k \in \mathbb{N}}$ generates $(\sigma, \hat{\nu}) \in \mathcal{G} \mathcal{D} \mathcal{M}_{\mathcal{F}}^{u_{D}}\left(\Omega ; \mathbb{R}^{m \times n}\right)$, it is bounded in $L^{1}\left(\Omega, \mathbb{R}^{m \times n}\right)$. The Dirichlet boundary condition on $\Gamma_{D}$ permits an application of the Poincaré inequality in the form

$$
\int_{\Omega}\left|u_{k}(x)\right| \mathrm{d} x \leq C\left(\int_{\Omega}\left|\nabla u_{k}(x)\right| \mathrm{d} x+\left|\int_{\Gamma_{D}} u_{D} \mathrm{~d} S\right|\right)
$$


and thus yields that $\left\{u_{k}\right\}_{k \in \mathbb{N}}$ is bounded in $W_{u_{D}}^{1,1}\left(\Omega ; \mathbb{R}^{m}\right)$ and therefore in $W^{1, \mu}\left(\Omega ; \mathbb{R}^{m}\right)$. Hence, there is a subsequence (not relabeled) converging weakly* in $W^{1, \mu}\left(\Omega ; \mathbb{R}^{m}\right)$ to some $u \in W^{1, \mu}\left(\Omega ; \mathbb{R}^{m}\right)[22]$, Theorem 6 . By definition of weak* convergence in $W^{1, \mu}\left(\Omega ; \mathbb{R}^{m}\right)$, this means that $u_{k} \rightarrow u$ strongly in $L^{1}\left(\Omega ; \mathbb{R}^{m}\right)$ and $\nabla u_{k} \rightarrow \bar{D} u$ weakly* in $M\left(\bar{\Omega} ; \mathbb{R}^{m \times n}\right)$. Formula (2.5) then follows by comparing (1.9) (with the obvious modification of using $\bar{D} u$ rather than $D u$, and allowing for test functions $\phi \in C(\bar{\Omega}))$ and (1.6) component-wise for $s=\left\{s_{j k}\right\}$, with $1 \leq j \leq m$ and $1 \leq k \leq n$. The fact that $u=u_{D}$ on $\Gamma_{D}$ follows from the weak* continuity of the trace operator $\bar{T}[22]$, Theorem 2 (ii).

Now let us discuss a suitable relaxation of the problem (2.3). We take a subalgebra $\mathcal{F}$ of bounded continuous functions on $\mathbb{R}^{m \times n}$ such that

$$
\tilde{W}(x, \cdot) \in \mathcal{F}
$$

we recall that $\mathcal{F}$ contains all functions where all radial limits exist, as a compactification by a sphere or finer is considered. We extend the previous notation slightly to accommodate for spatially inhomogeneous functions by writing $\tilde{W}(x, s):=\frac{W(x, s)}{1+|s|}$.

The relaxed problem then reads as follows:

$$
\begin{gathered}
\operatorname{minimise} \bar{I}(u, \bar{D} u, \sigma, \hat{\nu}):=\int_{\bar{\Omega}} \int_{\beta_{\mathcal{F}} \mathbb{R}^{m \times n}} \tilde{W}(x, s) \hat{\nu}_{x}(\mathrm{~d} s) \sigma(\mathrm{d} x)-\int_{\Omega} f(x) \cdot u(x) \mathrm{d} x \\
\text { among }(u, \bar{D} u) \in W^{1, \mu}\left(\Omega ; \mathbb{R}^{m}\right), \bar{T}(u, \bar{D} u)=u_{D} \text { on } \Gamma_{D}, \\
\text { and }(\sigma, \hat{\nu}) \in \mathcal{G} \mathcal{D} \mathcal{M}_{\mathcal{F}}^{u_{D}}\left(\Omega ; \mathbb{R}^{m \times n}\right), \bar{D} u \text { satisfies }(2.5) .
\end{gathered}
$$

Proposition 2.3. There is a constant $C=C(\Omega)>0$ depending on the domain $\Omega$ such that if

$$
\|f\|_{L^{n}\left(\Omega ; \mathbb{R}^{m}\right)}<C(\Omega)
$$

then a minimiser of (2.8) exists. Furthermore, the minimum of (2.8) equals the infimum of $(2.3)$. If $\left\{u_{k}\right\}_{k \in \mathbb{N}} \subset$ $W_{u_{D}}^{1,1}\left(\Omega ; \mathbb{R}^{m}\right)$ is an infimising sequence of (2.3), then a subsequence generates (in the sense (1.8)) a minimiser of (2.8). Moreover, any minimiser of (2.8) is generated by an infimising sequence of (2.3).

Proof. We first show that $\inf I \geq \inf \bar{I}$. Let $\left\{u_{k}\right\}_{k \in \mathbb{N}} \subset W_{u_{D}}^{1,1}\left(\Omega ; \mathbb{R}^{m}\right)$ be an infimising sequence of (2.3). Obviously inf $I<\infty$. Thus, there exists $K>0$ so that the following estimate holds (we employ the coercivity assumption (2.1) on $W$ together with Young's inequality with $q:=\frac{n}{n-1}$ in the second inequality and the Sobolev embedding [9], Thm. 5.6.2, in the third inequality, with $\left.C_{1}=C_{1}(\Omega)\right)$.

$$
\begin{aligned}
K & >\int_{\Omega} W\left(x, \nabla u_{k}(x)\right) \mathrm{d} x-\int_{\Omega} f(x) \cdot u_{k}(x) \mathrm{d} x \\
& \geq \alpha \int_{\Omega}\left|\nabla u_{k}(x)\right| \mathrm{d} x-\alpha|\Omega|-\left\|u_{k}\right\|_{L^{q}\left(\Omega ; \mathbb{R}^{m}\right)}\|f\|_{L^{n}\left(\Omega ; \mathbb{R}^{m}\right)} \\
& \geq \alpha \int_{\Omega}\left|\nabla u_{k}(x)\right| \mathrm{d} x-\alpha|\Omega|-C_{1}\left\|u_{k}\right\|_{W^{1,1}\left(\Omega ; \mathbb{R}^{m}\right)}\|f\|_{L^{n}\left(\Omega ; \mathbb{R}^{m}\right)} .
\end{aligned}
$$

Finally, using the Poincaré inequality (2.6) for the first term on the right, we obtain that $\left(C(\Omega)-\|f\|_{L^{n}\left(\Omega ; \mathbb{R}^{m}\right)}\right)$ $\left\|u_{k}\right\|_{W^{1,1}\left(\Omega ; \mathbb{R}^{m}\right)}$ is bounded from above, where $C(\Omega)$ is the quotient of the Poincaré embedding constant and $\frac{C_{1}}{\alpha}$. Thus, since the force is bounded by (2.9), it follows that $\sup _{k \in \mathbb{N}}\left\|u_{k}\right\|_{W^{1,1}\left(\Omega ; \mathbb{R}^{m}\right)}<\infty$. By the DiPerna-Majda result (1.6), $\left\{\nabla u_{k}\right\}_{k \in \mathbb{N}}$ then generates (up to a subsequence) $(\sigma, \hat{\nu}) \in \mathcal{G} \mathcal{D} \mathcal{M}_{\mathcal{F}}^{u_{D}}\left(\Omega ; \mathbb{R}^{m \times n}\right)$. At the same time we may suppose that $u_{k} \rightarrow u$ strongly in $L^{1}\left(\Omega ; \mathbb{R}^{m}\right)$ by compact embedding. Since $\left\{u_{k}\right\}_{k \in \mathbb{N}}$ is an infimising sequence, and the map $u \mapsto \int_{\Omega} f(x) \cdot u(x) \mathrm{d} x$ is sequentially continuous, (1.6) shows that $\inf I=\lim _{k \rightarrow \infty} I\left(u_{k}\right)=$ $\bar{I}(u, \bar{D} u, \sigma, \hat{\nu})$. To prove that $\inf I \geq \inf \bar{I}$, it remains to show that $(u, \bar{D} u, \sigma, \hat{\nu})$ is admissible for $(2.8)$. 
The sequence $\left\{u_{k}\right\}_{k \in \mathbb{N}}$ does not only converge strongly in $L^{1}\left(\Omega ; \mathbb{R}^{m}\right)$ to $u$, but is also bounded in $W^{1, \mu}\left(\Omega ; \mathbb{R}^{m}\right)$. Thus, it converges weakly* to $(u, \bar{D} u)$ in $W^{1, \mu}\left(\Omega ; \mathbb{R}^{m}\right)[22]$, Theorem 6 . In particular, for every $\phi \in C(\bar{\Omega})$,

$$
\lim _{k \rightarrow \infty} \int_{\bar{\Omega}} \phi(x) \nabla u_{k}(x) \mathrm{d} x=\int_{\bar{\Omega}} \phi(x) \bar{D} u(\mathrm{~d} x) .
$$

A comparison with (1.6) yields

$$
\int_{\bar{\Omega}} \phi(x) \bar{D} u(\mathrm{~d} x)=\int_{\bar{\Omega}} \phi(x) \int_{\beta_{\mathcal{F}} \mathbb{R}^{m \times n}} \tilde{s} \hat{\nu}_{x}(\mathrm{~d} s) \sigma(\mathrm{d} x),
$$

i.e., $\bar{D} u=\int_{\beta_{\mathcal{F}} \mathbb{R}^{m \times n}} \tilde{s} \hat{\nu}_{x}(\mathrm{~d} s) \sigma$. Thus, $\bar{D} u$ is indeed given by (2.5) as required. The limit $u$ satisfies $u=u_{D}$ on $\Gamma_{D}$, since the trace operator $W^{1, \mu}\left(\Omega ; \mathbb{R}^{m}\right) \rightarrow M\left(\partial \Omega ; \mathbb{R}^{m}\right)$ is weak* continuous [22], Theorem 2 (ii). Thus, $(u, \bar{D} u, \sigma, \hat{\nu})$ is admissible for the minimisation problem (2.8), and we have shown that $\inf I=\bar{I}(u, \bar{D} u, \sigma, \hat{\nu}) \geq$ $\inf \bar{I}$.

We sketch the proof of the existence of a minimiser of $\bar{I}$. To this end, let us consider a minimising sequence $\left\{\left(u_{j}, \bar{D} u_{j}, \sigma_{j}, \hat{\nu}_{j}\right)\right\}_{j \in \mathbb{N}}$ for $\bar{I}$. The alternative approach to DiPerna-Majda measures described in Section 1.3 suggests to write $\eta_{j} \cong\left(\sigma_{j}, \hat{\nu}_{j}\right)$ with $\eta_{j} \in G D M_{\mathcal{F}}^{u_{D}}\left(\Omega ; \mathbb{R}^{m \times n}\right)$ for any $j \in \mathbb{N}$. A modification of the preceding argument shows that generating sequences $\left\{\nabla u_{j}^{k}\right\}_{j, k \in \mathbb{N}}$, say, of $\left\{\eta_{j}\right\}_{j \in \mathbb{N}}$ are uniformly bounded in $L^{1}\left(\Omega ; \mathbb{R}^{m \times n}\right)$. Therefore $\left\{\eta_{j}\right\}_{j \in \mathbb{N}}$ form a weakly* compact subset of $M\left(\bar{\Omega} \times \beta_{\mathcal{F}} \mathbb{R}^{m \times n}\right)$, and we may suppose that $\eta_{j} \rightarrow \eta$ weakly* in $M\left(\bar{\Omega} \times \beta_{\mathcal{F}} \mathbb{R}^{m \times n}\right)$ as $j \rightarrow \infty$. Hence

$$
\lim _{j \rightarrow \infty} \lim _{k \rightarrow \infty} \int_{\bar{\Omega}} \phi(x) f\left(\nabla u_{j}^{k}(x)\right) \mathrm{d} x=\int_{\bar{\Omega} \times \beta_{\mathcal{F}} \mathbb{R}^{m \times n}} \phi(x) \tilde{f}(s) \eta(\mathrm{d} s, \mathrm{~d} x)
$$

for any $\tilde{f} \in \mathcal{F}$ and any $\phi \in C(\bar{\Omega})$. Since $C(\bar{\Omega})$ and $\mathcal{F}$ are separable, a diagonalisation argument yields a bounded sequence $\left\{w_{l}\right\}_{l \in \mathbb{N}} \subset W_{u_{D}}^{1,1}\left(\Omega ; \mathbb{R}^{m}\right)$ such that

$$
\lim _{l \rightarrow \infty} \int_{\bar{\Omega}} \phi(x) f\left(\nabla w_{l}(x)\right) \mathrm{d} x=\int_{\bar{\Omega} \times \beta_{\mathcal{F}} \mathbb{R}^{m \times n}} \phi(x) \tilde{f}(s) \eta(\mathrm{d} s, \mathrm{~d} x) .
$$

Moreover, $w_{k} \rightarrow u$ strongly in $L^{1}\left(\Omega ; \mathbb{R}^{m}\right)$ as $k \rightarrow \infty$, and $w_{k} \stackrel{\star}{\rightarrow}(u, \bar{D} u) \in W^{1, \mu}\left(\Omega ; \mathbb{R}^{m}\right)$. Altogether, $(u, \bar{D} u, \eta) \cong(u, \bar{D} u, \sigma, \hat{\nu})$ solves $(2.8)$.

Finally, we need to show the agreement of the arguments of $\inf I$ and $\inf \bar{I}$. Suppose there is $(v, \bar{D} v, \pi, \hat{\mu}) \in$ $W^{1, \mu}\left(\Omega ; \mathbb{R}^{m}\right) \times \mathcal{G} \mathcal{D} \mathcal{M}_{\mathcal{F}}^{u_{D}}\left(\Omega ; \mathbb{R}^{m \times n}\right)$ with $\bar{D} v=\int_{\beta_{\mathcal{F}} \mathbb{R}^{m \times n}} \tilde{s} \hat{\mu}_{x}(\mathrm{~d} s) \pi$ and $\bar{T}(v, \bar{D} v)=u_{D}$ on $\Gamma_{D}$ such that $\bar{I}(v, \bar{D} v, \pi, \hat{\mu})<\inf I$. Then, by the definition of $\mathcal{G} \mathcal{D} \mathcal{M}_{\mathcal{F}}^{u_{D}}\left(\Omega ; \mathbb{R}^{m \times n}\right)$, we infer that there is $\left\{v_{k}\right\}_{k \in \mathbb{N}} \subset$ $W_{u_{D}}^{1,1}\left(\Omega ; \mathbb{R}^{m}\right)$ such that $\left\{\nabla v_{k}\right\}_{k \in \mathbb{N}}$ generates $(\pi, \hat{\mu})$. As before we can show that $\left\{v_{k}\right\}_{k \in \mathbb{N}}$ is uniformly bounded in $W_{u_{D}}^{1,1}\left(\Omega ; \mathbb{R}^{m}\right)$ and we may suppose that $v_{k} \stackrel{\star}{\rightarrow}(w, \bar{D} w) \in W^{1, \mu}\left(\Omega ; \mathbb{R}^{m}\right)$ and $v_{k} \rightarrow w$ strongly in $L^{1}\left(\Omega ; \mathbb{R}^{m}\right)$. Then we obtain $\bar{D} v=\bar{D} w$. From this and from the fact that $\bar{T}(v, \bar{D} v)=\bar{T}(w, \bar{D} w)$ on $\Gamma_{D}$ we find that $(v, \bar{D} v)=(w, \bar{D} w)$. Hence, we arrive at $\lim _{k \rightarrow \infty} I\left(v_{k}\right)=\bar{I}(v, \bar{D} v, \pi, \hat{\mu})<\inf I$, i.e., for some $k \in \mathbb{N}$, we find $I\left(v_{k}\right)<\inf I$, a contradiction.

\section{Evolution}

We now consider an arbitrary, but fixed time interval $[0, T]$ and investigate the evolution of the material during this time. The evolution will be triggered by changes in the external force $f$. Energy may be dissipated during the evolution. We follow Mielke and co-workers $[15,17,18]$ in introducing a dissipation distance. At present, a detailed understanding of the atomistic evolution, which leads to the dissipation, is lacking. We thus adopt the common viewpoint that one has to resort to a scalar phenomenological model of the dissipation. 
It is then natural to define the (mesoscopic) dissipation distance between two DiPerna-Majda measures $\eta_{1}, \eta_{2} \in$ $G D M_{\mathcal{F}}^{u_{D}}\left(\Omega ; \mathbb{R}^{m \times n}\right)$, as these measure record the microstructure. Changing the overall state of the system from $\eta_{1}$ to $\eta_{2}$ then costs the energy $D\left(\eta_{1}, \eta_{2}\right)$. We recall that $\eta \cong(\sigma, \hat{\nu})$. It turns out to be advantageous for the rigorous analysis to have the dissipation $D$ depend on $\hat{\nu}$ and on the absolutely continuous part of $\sigma$. This is not a severe restriction, as the DiPerna-Majda measures with $\sigma$ being absolutely continuous with respect to the Lebesgue measure are dense in all DiPerna-Majda measures in the weak ${ }^{*}$ topology.

For the Young measure part $\hat{\nu}$, inspired by [17], we assume that there is $L \in \mathbb{N}$ and a bounded mapping $\Lambda: \mathbb{R}^{m \times n} \rightarrow \mathbb{R}^{L}$ which measures the contribution to the dissipation. Moreover, we use the following notation

$$
\operatorname{Id} \bullet \eta:=\int_{\beta_{\mathcal{F}} \mathbb{R}^{m \times n}} \frac{s}{1+|s|} \hat{\nu}_{x}(\mathrm{~d} s) \sigma .
$$

We summarise the assumptions on the dissipation.

Definition 3.1. The dissipation $D$ has to satisfy the following conditions.

(1) The triangle inequality is valid for $D$. That is, for any three internal states $\eta_{1}, \eta_{2}, \eta_{3}$, it holds that

$$
D\left(\eta_{1}, \eta_{3}\right) \leq D\left(\eta_{1}, \eta_{2}\right)+D\left(\eta_{2}, \eta_{3}\right)
$$

(2) We suppose that there is $L \in \mathbb{N}$ and a continuous bounded mapping $\Lambda: \mathbb{R}^{m \times n} \rightarrow \mathbb{R}^{L}$ such that $\Lambda_{j} \in \mathcal{F}$ for $1 \leq j \leq L$ such that the mesoscopic order parameter $\lambda$ associated with the system configuration described by $(u, \bar{D} u, \sigma, \hat{\nu})$ is given by the formula

$$
\lambda:=\int_{\beta_{\mathcal{F}} \mathbb{R}^{m \times n}} \Lambda(s) \hat{\nu}_{x}(\mathrm{~d} s) \sigma,
$$

which means that $\lambda \in M\left(\bar{\Omega} ; \mathbb{R}^{L}\right)$ is a measure such that, for all $g \in C(\bar{\Omega})$,

$$
\int_{\bar{\Omega}} g(x) \lambda(\mathrm{d} x)=\int_{\bar{\Omega}} \int_{\beta_{\mathcal{F}} \mathbb{R}^{m \times n}} \Lambda(s) \hat{\nu}_{x}(\mathrm{~d} s) g(x) \sigma(\mathrm{d} x) .
$$

Specifically, we write

$$
D\left(\eta_{1}, \eta_{2}\right)=\left\|\lambda_{1}-\lambda_{2}\right\|_{M\left(\bar{\Omega} ; \mathbb{R}^{L}\right)} .
$$

The definition (3.4) satisfies the triangle inequality (3.2).

Remark 3.2. (i) Writing $\alpha \in M\left(\bar{\Omega} ; \mathbb{R}^{L}\right)$ means that for any Borel measurable set $\omega \subset \Omega$, we have $\alpha(\omega)=$ $\left(\alpha_{1}(\omega), \ldots, \alpha_{L}(\omega)\right) \in \mathbb{R}^{L}$. Then we equip $\mathbb{R}^{L}$ by a norm $\|\cdot\|_{L}$. The $R^{L}$-valued total variation of $\alpha$, $|\alpha|$, is naturally defined as $|\alpha|=\left(\left|\alpha_{1}\right|, \ldots,\left|\alpha_{L}\right|\right)$.

(ii) If $\alpha \in M\left(\bar{\Omega} ; \mathbb{R}^{L}\right)$ is absolutely continuous with respect to the Lebesgue measure with the density $x \mapsto$ $\alpha(x) \in \mathbb{R}^{L}$, then it holds that

$$
\|\alpha\|_{M\left(\bar{\Omega} ; \mathbb{R}^{L}\right)}=\int_{\Omega}\left\|\alpha^{+}(x)+\alpha^{-}(x)\right\|_{L} \mathrm{~d} x=\|\alpha\|_{L^{1}\left(\Omega ; \mathbb{R}^{L}\right)} .
$$

Now we are in a position to define the set of admissible configurations. Each such configuration will be written as $q:=(u, \bar{D} u, \eta, \lambda)$. The set of admissible configurations is then

$$
\begin{gathered}
Q:=\left\{q=(u, \bar{D} u, \eta, \lambda) \in W^{1, \mu}\left(\Omega ; \mathbb{R}^{m}\right) \times G D M_{\mathcal{F}}^{u_{D}}\left(\Omega ; \mathbb{R}^{m \times n}\right) \times M\left(\bar{\Omega} ; \mathbb{R}^{L}\right)\right. \\
\left.D u=\operatorname{Id} \bullet \eta, \lambda \text { given by }(3.3), \text { and } \bar{T}(u, \bar{D} u)=u_{D} \text { on } \Gamma_{D}\right\}
\end{gathered}
$$


For convenience, we write

$$
D\left(q_{1}, q_{2}\right):=D\left(\eta_{1}, \eta_{2}\right)
$$

For a process $q:[0, T] \rightarrow Q$ and a given time interval $\left[t_{1}, t_{2}\right] \subset[0, T]$, the temporal dissipation is given by

$$
\operatorname{Diss}\left(q,\left[t_{1}, t_{2}\right]\right):=\sup _{L \in \mathbb{N}}\left\{\sum_{l=1}^{L} D\left(\eta\left(\tau_{l-1}\right), \eta\left(\tau_{l}\right)\right) \mid t_{1}=\tau_{0}<\tau_{2}<\cdots<\tau_{L}=t_{2}\right\} .
$$

It is natural to expect the evolution of $q$ to depend on applied external load $f$. The interesting case it that of a load $f$ varying over the given time interval $[0, T]$. In the below, we restrict ourselves to an external loading which depends continuously on time, $f \in W^{1,1}\left([0, T] ; L^{p}\left(\Omega ; \mathbb{R}^{m}\right)\right)$ with $p>n$. With $q=(u, \eta, \lambda)$, we shall write

$$
F(q):=\int_{\Omega} f(x, t) \cdot u(x) \mathrm{d} x
$$

for the contribution of the external load to the Gibbs energy. The power of the external load is then measured by

$$
\dot{F}(t, q)=\int_{\Omega} \frac{\partial f(x, t)}{\partial t} \cdot u(x) \mathrm{d} x
$$

We remind ourselves of the notation $\tilde{W}(x, s):=\frac{W(x, s)}{1+|s|}$. The Gibbs stored energy $E(t, q)$ is defined as

$$
E(t, q):=\int_{\bar{\Omega} \times \beta_{\mathcal{F}} \mathbb{R}^{m \times n}} \tilde{W}(x, s) \eta(\mathrm{d} s \mathrm{~d} x)-\int_{\Omega} f(x, t) \cdot u(x) \mathrm{d} x .
$$

At present, it seems not feasible to prove the existence of an energetic solution with the energy (3.8). We suppose that the measure $\lambda \in M\left(\bar{\Omega} ; \mathbb{R}^{L}\right)$ introduced in (3.3) is absolutely continuous with respect to the Lebesgue measure on $\Omega$. We identify it with its density $x \mapsto \lambda(x)$. Moreover, we will require that $\lambda$, which is by definition integrable, belongs even to $W^{1,2}\left(\Omega ; \mathbb{R}^{L}\right)$; see [17] for a similar regularisation, and a justification. Let $\varrho>0$; we then consider

$$
\Gamma_{\varrho}(t, q):=\int_{\bar{\Omega} \times \beta_{\mathcal{F}} \mathbb{R}^{m \times n}} \tilde{W}(x, s) \eta(\mathrm{d} s \mathrm{~d} x)+\varrho\|\lambda(x)\|_{W^{1,2}\left(\Omega ; \mathbb{R}^{L}\right)}-\int_{\Omega} f(x, t) \cdot u(x) \mathrm{d} x .
$$

Finally, we set

$$
\Gamma(t, q)= \begin{cases}\Gamma_{\varrho}(t, q) & \text { if } q \in Q \text { and } \lambda \in W^{1,2}\left(\Omega ; \mathbb{R}^{L}\right) \\ +\infty & \text { otherwise. }\end{cases}
$$

Notice that (3.10) excludes states of the system in which $\lambda$ is a measure which is not absolutely continuous with respect to the Lebesgue measure with fairly regular density. In particular, Remark 3.2 (ii) applies and (3.4) can be written as $D\left(\eta_{1}, \eta_{2}\right)=\left\|\lambda_{1}-\lambda_{2}\right\|_{L^{1}\left(\Omega ; \mathbb{R}^{L}\right)}$.

In this context, the notion of a solution to this energetic model can be stated as follows (see [17,18]; we merely repeat Def. 1.3 for the reader's convenience).

Definition 3.3. Given $q_{0} \in Q$, we say that the process $q:[0, T] \rightarrow Q$ is a solution to the problem under consideration if the following conditions hold:

(1) $(u, \bar{D} u) \in L^{\infty}\left(0, T ; W^{1, \mu}\left(\Omega ; \mathbb{R}^{m}\right)\right)$.

(2) $\lambda \in B V\left(0, T ; L^{1}\left(\Omega ; \mathbb{R}^{L}\right)\right)$.

(3) Global stability: For every $t \in[0, T]$, the process is stable in the global sense,

$$
\Gamma(t, q(t)) \leq \Gamma(t, \tilde{q})+D(q(t), \tilde{q}) \text { for every } \tilde{q} \in Q \text {. }
$$


(4) Energy inequality: For every $0 \leq t_{1} \leq t_{2} \leq T$, we have

$$
\Gamma\left(t_{1}, q\left(t_{1}\right)\right)+\operatorname{Diss}\left(q,\left[t_{1}, t_{2}\right]\right) \leq \Gamma\left(t_{2}, q\left(t_{2}\right)\right)-\int_{t_{1}}^{t_{2}} \dot{F}(t, q(t)) \mathrm{d} t
$$

(5) Initial condition: $q(0)=q_{0}$ and $\Gamma(0, q(0))<\infty$.

We point out that this notion of a solution is based on global stability. This means that a state $q$ is stable if and only if the potential reduction of the Gibbs energy at another state $\tilde{q} \in Q$ is not larger than the dissipation $D(q, \tilde{q})$. We refer the reader to [18] for a discussion. It is convenient to denote the set of globally stable states at a time instant $t$ by $S(t)$,

$$
S(t):=\{q \in Q \mid \Gamma(t, q) \leq \Gamma(t, \tilde{q})+D(q, \tilde{q}) \text { for every } \tilde{q} \in Q\} .
$$

The following statement is technical, but crucial for the arguments proving the existence of an energetic solution.

Proposition 3.4. Consider a sequence $\left\{\left(t_{k}, q_{k}\right)\right\}_{k \in \mathbb{N}}$ with $q_{k} \in S\left(t_{k}\right)$ such that $t_{k} \rightarrow t$, and $q_{k} \rightarrow q$ weakly* in the sense that $\left(u_{k}, \bar{D} u_{k}\right) \rightarrow(u, \bar{D} u)$ weakly* in $W^{1, \mu}\left(\Omega ; \mathbb{R}^{m}\right)$, while $\eta_{k} \rightarrow \eta$ weakly* in $C\left(\bar{\Omega} \times \beta_{\mathcal{F}} \mathbb{R}^{m \times n}\right)^{*} \cap$ $G D M_{\mathcal{F}}^{u_{D}}\left(\Omega ; \mathbb{R}^{m \times n}\right)$ and $\lambda_{k} \rightarrow \lambda$ weakly in $W^{1,2}\left(\Omega ; \mathbb{R}^{L}\right)$ as $k \rightarrow \infty$. For the external forcing $f$, we assume that, for $p>n, f \in W^{1,1}\left([0, T] ; L^{p}\left(\Omega ; \mathbb{R}^{m}\right)\right)$ and furthermore that, for a constant $C(\Omega)$ specified in the proof, $\|f(t)\|_{L^{n}\left(\Omega ; \mathbb{R}^{m}\right)} \leq C(\Omega)$ for all $t \in[0, T]$. Then $q(t) \in S(t)$ and $\Gamma\left(t_{k}, q_{k}\right) \rightarrow \Gamma(t, q)$ as $k \rightarrow \infty$.

Proof. Our first goal is to show that $\Gamma(t, q) \leq \lim _{k \rightarrow \infty} \Gamma\left(t_{k}, q_{k}\right)$. The argument uses that $\eta_{k} \rightarrow \eta$ weakly* in $C\left(\bar{\Omega} \times \beta_{\mathcal{F}} \mathbb{R}^{m \times n}\right)^{*}$ as $k \rightarrow \infty$. As $\tilde{W}$ is continuous and $C(\bar{\Omega}) \otimes C\left(\beta_{\mathcal{F}} \mathbb{R}^{m \times n}\right)$ is dense in $C\left(\bar{\Omega} \times \beta_{\mathcal{F}} \mathbb{R}^{m \times n}\right)$, this implies

$$
\int_{\bar{\Omega} \times \beta_{\mathcal{F}} \mathbb{R}^{m \times n}} \tilde{W}(x, s) \eta_{k}(\mathrm{~d} s \mathrm{~d} x) \rightarrow \int_{\bar{\Omega} \times \beta_{\mathcal{F}} \mathbb{R}^{m \times n}} \tilde{W}(x, s) \eta(\mathrm{d} s \mathrm{~d} x) .
$$

Since $u_{k} \rightarrow u$ strongly in $L^{r}\left(\Omega ; \mathbb{R}^{m}\right)$ with $1 \leq r<\frac{n}{n-1}$ for $k \rightarrow \infty[22]$, Theorem 5 , and $f \in W^{1,1}\left([0, T] ; L^{p}\left(\Omega ; \mathbb{R}^{m}\right)\right)$ with $p>n$, it is immediate that

$$
\lim _{k \rightarrow \infty} \int_{\Omega} f\left(t_{k}, x\right) \cdot u_{k}(x) \mathrm{d} x=\int_{\Omega} f(t, x) \cdot u(x) \mathrm{d} x
$$

Relations (3.13), (3.14) and the lower semicontinuity of the norm (here $\|\lambda\|_{W^{1,2}\left(\Omega ; \mathbb{R}^{L}\right)}$ ) in the weak topology taken together prove that

$$
\Gamma(t, q) \leq \lim _{k \rightarrow \infty} \Gamma\left(t_{k}, q_{k}\right)
$$

Now for the stability of $q$. We use the main properties of $D$. Namely, for arbitrary $\tilde{q} \in Q$, the triangle inequality (3.2) for $D$ and the expression of $D$ given in (3.4) yield (note that $\lambda_{k} \rightarrow \lambda$ strongly in $L^{1}\left(\Omega ; \mathbb{R}^{L}\right)$ by compact embedding of $W^{1,2}\left(\Omega ; \mathbb{R}^{L}\right)$ in $\left.L^{1}\left(\Omega ; \mathbb{R}^{L}\right)\right)$

$$
\lim _{k \rightarrow \infty}\left|D\left(q_{k}, \tilde{q}\right)-D(q, \tilde{q})\right| \leq \lim _{k \rightarrow \infty} D\left(q_{k}, q\right)=0
$$

Altogether, the lower semicontinuity (3.15), the stability of $q_{k}$ at time $t_{k}$ and (3.16) show the global stability $q$,

$$
\Gamma(t, q) \leq \lim _{k \rightarrow \infty} \Gamma\left(t_{k}, q_{k}\right) \leq \lim _{k \rightarrow \infty} \Gamma\left(t_{k}, \tilde{q}\right)+D\left(q_{k}, \tilde{q}\right)=\Gamma(t, \tilde{q})+D(q, \tilde{q})
$$

Equation (3.17) proves the claimed stability; for $\tilde{q}:=q$, it also yields, together with (3.15), the continuity $\Gamma(t, q)=\lim _{k \rightarrow \infty} \Gamma\left(t_{k}, q_{k}\right)$. 
The only open claim so far is that $q \in S(t)$. Since the global stability of $q$ has just been proved, it remains to prove that $q \in Q$. An argument similar to the one used in the proof of Proposition 2.3 shows that for $r:=\frac{n}{n-1}$

$$
\Gamma\left(t_{k}, q_{k}\right) \geq \alpha \sigma_{k}(\bar{\Omega})-\alpha|\Omega|-\left\|f\left(t_{k}, \cdot\right)\right\|_{L^{n}\left(\Omega ; \mathbb{R}^{m}\right)}\left\|u_{k}\right\|_{L^{r}\left(\Omega ; \mathbb{R}^{m}\right)} .
$$

As $\Gamma\left(t_{k}, q_{k}\right)$ converges to $\Gamma(t, q)$ as shown above in (3.17), we may suppose that for $k \in \mathbb{N}$ large enough $\Gamma(t, q)+1 \geq \alpha \sigma_{k}(\bar{\Omega})-\alpha|\Omega|-\left\|f\left(t_{k}, \cdot\right)\right\|_{L^{n}\left(\Omega ; \mathbb{R}^{m}\right)}\left\|u_{k}\right\|_{L^{r}\left(\Omega ; \mathbb{R}^{m}\right)}$. In other words,

$$
\Gamma(t, q)+1 \geq \lim _{j \rightarrow \infty} \int_{\Omega} \alpha\left|\nabla u_{k}^{j}(x)\right| \mathrm{d} x-\alpha|\Omega|-C\left\|f\left(t_{k}, \cdot\right)\right\|_{L^{n}\left(\Omega ; \mathbb{R}^{m}\right)}\left\|u_{k}^{j}\right\|_{W^{1,1}\left(\Omega ; \mathbb{R}^{m}\right)},
$$

where $\left\{u_{k}^{j}\right\}_{j \in \mathbb{N}} \subset W_{u_{D}}^{1,1}\left(\Omega ; \mathbb{R}^{m}\right)$ is a generating sequence of $\left(\sigma_{k}, \hat{\nu}_{k}\right)$ and $C$ is given by the Sobolev embedding [9], Theorem 5.6.2. With the Poincaré inequality (2.6), it follows as in the proof of Proposition 2.3 that

$$
\left(C(\Omega)-\left\|f\left(t_{k}, \cdot\right)\right\|_{L^{n}\left(\Omega ; \mathbb{R}^{m}\right)}\right)\left\|u_{k}^{j}\right\|_{W^{1,1}\left(\Omega ; \mathbb{R}^{m}\right)}
$$

is bounded from above, and then by assumption $\left\|u_{k}^{j}\right\|_{W^{1,1}\left(\Omega ; \mathbb{R}^{m}\right)}$ is bounded. A diagonalisation argument together with the assumption $\eta_{k} \rightarrow \eta$ for $k \rightarrow \infty$ shows that $\eta \in G D M_{\mathcal{F}}^{u_{D}}\left(\Omega ; \mathbb{R}^{m \times n}\right)$.

This gives us a uniform bound on all sequences generating $\left\{\eta_{k}\right\}_{k \in \mathbb{N}}$. Since $\eta_{k} \rightarrow \eta$ for $k \in \mathbb{N}$, the rest of the proof is analogous to the proof of Proposition 2.3. Notice that $\eta_{k} \rightarrow \eta$ implies that $\lambda$ satisfies (3.3) with $\eta \cong(\sigma, \hat{\nu})$.

\subsection{Incremental problems}

With these preparations in place, it is not hard to harvest the results. Namely, the argument proving the existence of an energetic solution now follows the established path of analysing the incremental time step problem, and then taking the passage to the limit as the time increment goes to zero. We now turn our attention to the incremental problem. Let us recall that $T$ is an arbitrary, but finite final time. It is not restrictive to choose the time step $\tau$ such that $N:=\frac{T}{\tau} \in \mathbb{N}$. For a given initial condition $q_{\tau}^{0}=q_{0}$, it is natural to define $q_{\tau}^{k}$ for $k=1, \ldots, N$ as a solution to the problem

$$
\min _{q \in Q} \Gamma(k \tau, q)+D\left(q_{\tau}^{k-1}, q\right) .
$$

We introduce a piecewise constant interpolation $q_{\tau}$ by setting $q_{\tau}(t):=q_{\tau}^{k}$ for $t \in((k-1) \tau, k \tau]$ and $k=$ $1, \ldots, \frac{T}{\tau}$. Analogously, $F_{\tau}(t, q):=F(k \tau, q)$ is a piecewise constant interpolation of $F$, and

$$
\Gamma_{\tau}(t, q):=\Gamma(k \tau, q) .
$$

Proposition 3.5. Let $f \in W^{1,1}\left(0, T ; L^{p}\left(\Omega ; \mathbb{R}^{n}\right)\right)$, for some $p>n$, with $\|f(t)\|_{L^{n}\left(\Omega ; \mathbb{R}^{m}\right)} \leq C(\Omega)$ for all $t \in[0, T]$, as in Proposition 3.4. Then the problem (3.18) has a solution $q_{\tau}(t)$ which is stable, i.e., for all $t \in[0, T]$ and for every $\tilde{q} \in Q$,

$$
\Gamma_{\tau}\left(t, q_{\tau}(t)\right) \leq \Gamma_{\tau}(t, \tilde{q})+D\left(q_{\tau}(t), \tilde{q}\right) .
$$

Moreover, for all $t_{1} \leq t_{2}$ from the set $\{k \tau\}_{k=0}^{N}$, the following discrete energy inequalities hold if one extends the definition of $q_{\tau}(t)$ by setting $q_{\tau}(t):=q_{0}$ if $t<0$.

$$
\begin{aligned}
-\int_{t_{1}}^{t_{2}} \dot{F}\left(t, q_{\tau}(t)\right) \mathrm{d} t & \leq \Gamma\left(t_{2}, q_{\tau}\left(t_{2}\right)\right)+\operatorname{Diss}\left(q_{\tau},\left[t_{1}, t_{2}\right]\right)-\Gamma\left(t_{1}, q_{\tau}\left(t_{1}\right)\right) \\
& \leq \int_{t_{1}}^{t_{2}} \dot{F}\left(t, q_{\tau}(t-\tau)\right) \mathrm{d} t .
\end{aligned}
$$


Proof. The existence of a solution to (3.18) follows from the coercivity of $G(q):=\Gamma(k \tau, q)+D\left(q_{\tau}^{k-1}, q\right)$. The weak* lower semicontinuity of $G$ implies the existence of a solution.

The stability estimate (3.19) follows from the minimising property of $q_{\tau}^{k}$ and the properties of $D$. Indeed, by minimality of $q_{\tau}^{k}$,

from which we infer that

$$
\Gamma\left(k \tau, q_{\tau}^{k}\right)+D\left(q_{\tau}^{k-1}, q_{\tau}^{k}\right) \leq \Gamma(k \tau, \tilde{q})+D\left(q_{\tau}^{k-1}, \tilde{q}\right),
$$

$$
\Gamma\left(k \tau, q_{\tau}^{k}\right) \leq \Gamma(k \tau, \tilde{q})+D\left(q_{\tau}^{k-1}, \tilde{q}\right)-D\left(q_{\tau}^{k-1}, q_{\tau}^{k}\right) .
$$

However, the structure of the metric (see Rem. 3.2) implies that

$$
D\left(q_{\tau}^{k-1}, \tilde{q}\right)-D\left(q_{\tau}^{k-1}, q_{\tau}^{k}\right) \leq D\left(q_{\tau}^{k}, \tilde{q}\right),
$$

from which (3.19) follows.

Next, we demonstrate the validity of the energy inequality (3.20), following the arguments in [18]. For this part, let us test the stability of $q_{\tau}^{k-1}$ with $\tilde{q}:=q_{\tau}^{k}$. This gives

$$
\begin{aligned}
\Gamma\left((k-1) \tau, q_{\tau}^{k-1}\right) & \leq \Gamma\left((k-1) \tau, q_{\tau}^{k}\right)+D\left(q_{\tau}^{k-1}, q_{\tau}^{k},\right) \\
& =\Gamma\left(k \tau, q_{\tau}^{k}\right)+F\left(k \tau, q_{\tau}^{k}\right)-F\left((k-1) \tau, q_{\tau}^{k}\right)+D\left(q_{\tau}^{k-1}, q_{\tau}^{k}\right) .
\end{aligned}
$$

Suppose that $0 \leq k_{1} \leq k_{2} \leq N$ and that $t_{1}=k_{1} \tau$ and $t_{2}=k_{2} \tau$. A summation of $(3.22)$ over $k=k_{1}+1, \ldots, k_{2}$ yields

$$
\sum_{k=k_{1}+1}^{k_{2}}\left[F\left((k-1) \tau, q_{\tau}^{k}\right)-F\left(k \tau, q_{\tau}^{k}\right)\right] \leq \Gamma\left(k_{2} \tau, q_{\tau}^{k_{2}}\right)-\Gamma\left(k_{1} \tau, q_{\tau}^{k_{1}}\right)+\sum_{k=k_{1}+1}^{k_{2}} D\left(q_{\tau}^{k-1}, q_{\tau}^{k}\right) .
$$

We rewrite this inequality in terms of $q_{\tau}$ to see that it is the first inequality in (3.20),

$$
\begin{aligned}
-\int_{t_{1}}^{t_{2}} \dot{F}\left(t, q_{\tau}(t)\right) \mathrm{d} t & \leq \Gamma\left(k_{2} \tau, q_{\tau}^{k_{2}}\right)-\Gamma\left(k_{1} \tau, q_{\tau}^{k_{1}}\right)+\sum_{k=k_{1}+1}^{k_{2}} D\left(q_{\tau}^{k-1}, q_{\tau}^{k}\right) \\
& =\Gamma\left(k_{2} \tau, q_{\tau}^{k_{2}}\right)-\Gamma\left(k_{1} \tau, q_{\tau}^{k_{1}}\right)+\operatorname{Diss}\left(q_{\tau},\left[t_{1}, t_{2}\right]\right)
\end{aligned}
$$

(the explicit form of Diss $\left(q_{\tau},\left[t_{1}, t_{2}\right]\right)$ holds since we consider a step function). To prove the validity of the second inequality in (3.20), we rely on the minimality of $q_{\tau}^{k}$, when compared to as $\tilde{q}:=q_{\tau}^{k-1}$ in (3.21). That is,

$$
\begin{aligned}
\Gamma\left(k \tau, q_{\tau}^{k}\right)+D\left(q_{\tau}^{k-1}, q_{\tau}^{k}\right) & \leq \Gamma\left(k \tau, q_{\tau}^{k-1}\right) \\
& =\Gamma\left((k-1) \tau, q_{\tau}^{k-1}\right)+F\left((k-1) \tau, q_{\tau}^{k-1}\right)-F\left(k \tau, q_{\tau}^{k-1}\right) .
\end{aligned}
$$

Similarly as in the previous argument, a summation over $k=k_{1}+1, \ldots, k_{2}$ is employed to find that

$$
\Gamma\left(k_{2} \tau, q_{\tau}^{k_{2}}\right)-\Gamma\left(k_{1} \tau, q_{\tau}^{k_{1}}\right)+\sum_{k=k_{1}+1}^{k_{2}} D\left(q_{\tau}^{k-1}, q_{\tau}^{k}\right) \leq \sum_{k=k_{1}+1}^{k_{2}}\left[F\left((k-1) \tau, q_{\tau}^{k-1}\right)-F\left(k \tau, q_{\tau}^{k-1}\right)\right],
$$

so that

$$
\Gamma\left(k_{2} \tau, q_{\tau}^{k_{2}}\right)-\Gamma\left(k_{1} \tau, q_{\tau}^{k_{1}}\right)+\operatorname{Diss}\left(q_{\tau},\left[t_{1}, t_{2}\right]\right) \leq-\int_{t_{1}}^{t_{2}} \dot{F}\left(t, q_{\tau}(t-\tau)\right) \mathrm{d} t,
$$

which is the second inequality in (3.20).

The next proposition gives the a priori bounds needed to pass to the limit as the step size goes to zero. 
Proposition 3.6. Assume that $W$ satisfies the growth condition $(2.1)$. Let $f \in W^{1,1}\left(0, T ; L^{p}\left(\Omega ; \mathbb{R}^{m}\right)\right)$ with $p>n$ be such that there is a constant $C=C(\Omega, W)>0$ depending only on $\Omega$ and $W$ as specified in the proof with

$$
\max _{t \in[0, T]}\|f(t, \cdot)\|_{L^{p}\left(\Omega ; \mathbb{R}^{m}\right)}+\int_{0}^{T}\left\|\frac{\partial f}{\partial t}(t, \cdot)\right\|_{L^{p}\left(\Omega ; \mathbb{R}^{m}\right)} \mathrm{d} t \leq C(\Omega, W) .
$$

Then there is $\kappa \in \mathbb{R}$ such that

$$
\begin{gathered}
\left\|\left(u_{\tau}, \bar{D} u_{\tau}\right)\right\|_{L^{\infty}\left(0, T ; W^{1, \mu}\left(\Omega ; \mathbb{R}^{m}\right)\right)}<\kappa, \\
\left\|\lambda_{\tau}\right\|_{L^{\infty}\left(0, T ; W^{1,2}\left(\Omega ; \mathbb{R}^{L}\right)\right) \cap B V\left(0, T ; L^{1}\left(\Omega ; \mathbb{R}^{L}\right)\right)}<\kappa,
\end{gathered}
$$

and for $\hat{\Gamma}_{\tau}(t):=\Gamma_{\tau}\left(t, q_{\tau}(t)\right)$,

$$
\left\|\hat{\Gamma}_{\tau}\right\|_{B V(0, T)}<\kappa
$$

Proof. The growth condition (2.1) implies for $q \in Q$ (i.e., $\left.\eta \in G D M_{\mathcal{F}}^{u_{D}}\left(\Omega ; \mathbb{R}^{m \times n}\right)\right)$ the estimate

$$
\|\bar{D} u\|_{M\left(\bar{\Omega} ; \mathbb{R}^{m \times n}\right)} \leq \int_{\bar{\Omega} \times \beta_{\mathcal{F}} \mathbb{R}^{m \times n}} \frac{|s|}{1+|s|} \eta(\mathrm{d} s \mathrm{~d} x) \leq \frac{1}{\alpha} \int_{\bar{\Omega} \times \beta_{\mathcal{F}} \mathbb{R}^{m \times n}} \frac{W(x, s)+\alpha|\Omega|}{1+|s|} \eta(\mathrm{d} s \mathrm{~d} x) .
$$

Using the Poincaré-type inequality (1.13), we obtain

$$
\|(u, \bar{D} u)\|_{W^{1, \mu}\left(\Omega ; \mathbb{R}^{m}\right)} \leq C\left\|u_{D}\right\|_{M\left(\Gamma_{D} ; \mathbb{R}^{m}\right)}+\frac{C}{\alpha} \int_{\bar{\Omega} \times \beta_{\mathcal{F}} \mathbb{R}^{m \times n}} \frac{W(x, s)+\alpha|\Omega|}{1+|s|} \eta(\mathrm{d} s \mathrm{~d} x) .
$$

Hence,

$$
\frac{\alpha}{C}\|(u, \bar{D} u)\|_{W^{1, \mu}\left(\Omega ; \mathbb{R}^{m}\right)} \leq \alpha\left\|u_{D}\right\|_{M\left(\Gamma_{D} ; \mathbb{R}^{m}\right)}+\int_{\bar{\Omega} \times \beta_{\mathcal{F}} \mathbb{R}^{m \times n}} \tilde{W}(x, s) \eta(\mathrm{d} s \mathrm{~d} x)+C .
$$

Trivially, this implies

$$
\begin{aligned}
\varrho\|\lambda(x)\|_{W^{1,2}\left(\Omega ; \mathbb{R}^{L}\right)}+\frac{\alpha}{C}\|(u, \bar{D} u)\|_{W^{1, \mu}\left(\Omega ; \mathbb{R}^{m}\right)} \leq & \alpha\left\|u_{D}\right\|_{M\left(\Gamma_{D} ; \mathbb{R}^{m}\right)}+C \\
& +\int_{\bar{\Omega} \times \beta_{\mathcal{F}} \mathbb{R}^{m \times n}} \tilde{W}(x, s) \eta(\mathrm{d} s \mathrm{~d} x)+\varrho\|\lambda(x)\|_{W^{1,2}\left(\Omega ; \mathbb{R}^{L}\right)} .
\end{aligned}
$$

The right-hand side is the sum of a boundary term, a constant and, by (3.10) and (3.7), the Gibbs stored energy $\Gamma\left(k_{2} \tau, q_{\tau}^{k_{2}}\right)$ plus the energy related to the external loading. We use (3.24) with $k_{1}:=0$ to deduce that

$$
\begin{aligned}
\varrho\left\|\lambda_{\tau}^{k_{2}}\right\|_{W^{1,2}\left(\Omega ; \mathbb{R}^{L}\right)}+\frac{\alpha}{C}\left\|u_{\tau}^{k_{2}}\right\|_{W^{1, \mu}\left(\Omega ; \mathbb{R}^{m}\right)} \leq & \alpha\left\|u_{D}\right\|_{M\left(\Gamma_{D} ; \mathbb{R}^{m}\right)}+\Gamma\left(0, q^{0}\right)+C \\
& +\sum_{k=1}^{k_{2}}\left[F\left((k-1) \tau, q_{\tau}^{k-1}\right)-F\left(k \tau, q_{\tau}^{k-1}\right)\right] \\
& +F\left(k_{2} \tau, q_{\tau}^{k_{2}}\right) \\
= & \alpha\left\|u_{D}\right\|_{M\left(\Gamma_{D} ; \mathbb{R}^{m}\right)}+\Gamma\left(0, q^{0}\right)+C \\
& +\int_{\partial \Omega}\left[\sum_{k=1}^{k_{2}}\left(f_{\tau}^{k-1}-f_{\tau}^{k}\right) \cdot u_{\tau}^{k-1}+f_{\tau}^{k_{2}} \cdot u_{\tau}^{k_{2}}\right] .
\end{aligned}
$$


We set $U_{\tau}:=\max _{0 \leq k \leq N}\left\|\left(u_{\tau}^{k}, \bar{D} u_{\tau}^{k}\right)\right\|_{W^{1, \mu}\left(\Omega ; \mathbb{R}^{m}\right)}$ and $\Lambda_{\tau}:=\max _{0 \leq k \leq N}\left\|\lambda_{\tau}^{k}\right\|_{W^{1,2}\left(\Omega ; \mathbb{R}^{L}\right)}$. Then

$$
\begin{aligned}
\frac{1}{2}\left(\varrho \Lambda_{\tau}+\frac{\alpha}{C} U_{\tau}\right) \leq \alpha\left\|u_{D}\right\|_{M\left(\Gamma_{D} ; \mathbb{R}^{m}\right)}+ & \Gamma\left(0, q^{0}\right)+C \\
& +\left(\int_{0}^{T}\left\|\frac{\partial f}{\partial t}(t, \cdot)\right\|_{L^{p}\left(\Omega ; \mathbb{R}^{m}\right)} \mathrm{d} t+\max _{t \in[0, T]}\|f(t, \cdot)\|_{L^{p}\left(\Omega ; \mathbb{R}^{m}\right)}\right) U_{\tau} .
\end{aligned}
$$

The bound (3.25) with the choice $0<C(\Omega, W)<\frac{\alpha}{C}$ then proves (3.26) as well as the bound for $\left\|\lambda_{\tau}\right\|_{L^{\infty}\left(0, T ; W^{1,2}\left(\Omega ; \mathbb{R}^{L}\right)\right)}$ claimed in (3.27).

To prove the second bound for $\lambda_{\tau}$ in (3.27), we infer from (3.24) for $k_{1}:=0$ and $k_{2}:=N$ that

$$
\Gamma\left(T, q_{\tau}^{N}\right)-\Gamma\left(0, q^{0}\right) \leq U_{\tau} \int_{0}^{T}\left\|\frac{\partial f}{\partial t}(t, \cdot)\right\|_{L^{p}\left(\Omega ; \mathbb{R}^{m}\right)} \mathrm{d} t .
$$

From the bound (3.26) which we have just proved via (3.30), we see that the right-hand side of (3.31) is bounded independently of $\tau$. In particular, (3.20) yields for $D$ defined in (3.6) that

$$
\sum_{k=1}^{N} D\left(q_{\tau}^{k-1}, q_{\tau}^{k}\right)=\int_{\Omega} \sum_{k=1}^{N}\left\|\lambda_{\tau}^{k-1}(x)-\lambda_{\tau}^{k}(x)\right\|_{L} \mathrm{~d} x=\left\|\sum_{k=1}^{N}\right\| \lambda_{\tau}^{k-1}-\lambda_{\tau}^{k}\left\|_{L}\right\|_{L^{1}(\Omega)}
$$

is bounded independently of $\tau$. Thus the second bound $\left\|\lambda_{\tau}\right\|_{B V\left(0, T ; L^{1}\left(\Omega ; \mathbb{R}^{L}\right)\right)}<\kappa$ in (3.27) holds as well.

Finally, let us prove the bound (3.28). The energy inequalities (3.23)-(3.24) with $k_{2}:=k, k_{1}:=k-1$ and the triangle inequality (3.2) for $D$ imply

$$
\begin{aligned}
\left|\Gamma\left(k \tau, q_{\tau}^{k}\right)-\Gamma\left((k-1) \tau, q_{\tau}^{k-1}\right)\right| & \leq D\left(q_{\tau}^{k}, q_{\tau}^{k-1}\right) \\
& +\max \left\{\left|F\left((k-1) \tau, q_{\tau}^{k}\right)-F\left(k \tau, q_{\tau}^{k}\right)\right|,\left|F\left((k-1) \tau, q_{\tau}^{k-1}\right)-F\left(k \tau, q_{\tau}^{k-1}\right)\right|\right\} .
\end{aligned}
$$

The qualification of the force $f$ and (3.32) on $D$ imply the desired estimate (3.28).

\section{LIMIT PASSAGE FOR $\tau \rightarrow 0$}

We make one more assumption (see [17]). Namely, we require the following condition to hold for every $t \in[0, T]$ and for every $q_{j}=\left(u_{j}, \bar{D} u_{j}, \eta_{j}, \lambda_{j}\right) \in S(t)$, with $j=1,2$ :

$$
\text { if } \lambda_{1}=\lambda_{2} \text {, then }\left(u_{1}, \bar{D} u_{1}\right)=\left(u_{2}, \bar{D} u_{2}\right) \text {. }
$$

This condition is not inevitable; it is mainly a requirement on the map $\Lambda$ describing the dissipation mechanism; see [12] for how to proceed without this assumption.

Then we have the following proposition (see [17], Prop. 3.3).

Proposition 4.1. Let (4.1) hold. The requirements on the loading $f$ stated in Proposition 3.4 are assumed to hold. Let $q_{k}=\left(u_{k}, \bar{D} u_{k}, \eta_{k}, \lambda_{k}\right) \in S\left(t_{k}\right)$ with some $t_{k} \rightarrow t$ and $\lambda_{k} \rightarrow \lambda$ weakly in $W^{1,2}\left(\Omega ; \mathbb{R}^{L}\right)$. Then $u_{k} \rightarrow u$ in $L^{1}\left(\Omega ; \mathbb{R}^{m}\right)$.

Proof. Since $\left\{\left(u_{k}, \bar{D} u_{k}\right)\right\}_{k \in \mathbb{N}}$ is bounded in $W^{1, \mu}\left(\Omega ; \mathbb{R}^{m}\right)$ by $(3.26)$, we can extract a converging subsequence (not relabeled) in $L^{1}\left(\Omega ; \mathbb{R}^{m}\right)$. Moreover, $\left(u_{k}, \bar{D} u_{k}\right) \stackrel{\star}{\rightarrow}(u, \bar{D} u)$ weakly* in $W^{1, \mu}\left(\Omega ; \mathbb{R}^{m}\right)$, again by [22], Theorem 6. Furthermore, $\eta_{k} \stackrel{\star}{\rightarrow} \eta$ in $C\left(\bar{\Omega} \times \beta_{\mathcal{F}} \mathbb{R}^{m \times n}\right)^{*} \cap G D M_{\mathcal{F}}^{u_{D}}\left(\Omega ; \mathbb{R}^{m \times n}\right)$ for a suitable subsequence. Thus for any such subsequence (not relabeled) with weak limit $q$, say, it holds that $q \in S(t)$ by Proposition 3.4. 
Since the limit $u$ is by (4.1) unique, independently of the choice of the convergent subsequence of $\left\{u_{k}\right\}_{k \in \mathbb{N}}$, the entire sequence converges.

The following proposition uses the concept of nets from general topology. Here, we only briefly recall this notion, which generalises the notion of sequences. We refer the reader to [8] for more details. The set $\left\{x_{\xi}\right\} \xi \in \Xi \subset X$ is a net if the index set $\Xi$ is directed, i.e., it is partially ordered and has the property that any two elements have a common majorant. The net $\left\{x_{\xi}\right\}_{\xi \in \Xi}$ is said to be convergent to $x_{0}, \lim _{\xi \in \Xi} x_{\xi}=x_{0}$, if for every neighbourhood $U$ of $x_{0}$, there is $\xi_{0}$ such that $x_{\xi} \in U$ for all $\xi \succeq \xi_{0}$. The concept corresponding to subsequences are finer nets. A net $\left\{y_{\tilde{\xi}}\right\}_{\tilde{\xi} \in \tilde{\Xi}}$ is a finer net than $\left\{x_{\xi}\right\}_{\xi \in \Xi}$ if there is a mapping $\phi: \tilde{\Xi} \rightarrow \Xi$ such that for any $\tilde{\xi} \in \tilde{\Xi}$, it holds that $y_{\tilde{\xi}}=x_{\phi(\xi)}$ and furthermore for any $\xi \in \Xi$ there is $\tilde{\xi} \in \tilde{\Xi}$ such that $\phi(\tilde{\xi}) \succeq \xi$ if $\tilde{\xi} \succeq \tilde{\xi}$. Compact sets enjoy the property that every net possesses a finer net that converges.

Proposition 4.2. We assume $f \in W^{1,1}\left(0, T ; L^{p}\left(\Omega ; \mathbb{R}^{n}\right)\right)$, for some $p>n$, with $\|f(t)\|_{L^{n}\left(\Omega ; \mathbb{R}^{m}\right)} \leq C(\Omega)$ for all $t \in[0, T]$, as in Proposition 3.4. In addition, (3.25) has to hold. Let $\left\{q_{\tau_{k}}\right\}$ be a sequence of solutions to (3.18), indexed by vanishing step-sizes $\tau_{k}=\frac{T}{2^{k}}$. Then there is its subsequence (not relabeled) and $q:[0, T] \rightarrow$ $W^{1, \mu}\left(\Omega ; \mathbb{R}^{m}\right) \times G D M_{\mathcal{F}}^{u_{D}}\left(\Omega ; \mathbb{R}^{m \times n}\right) \times L^{1}\left(\Omega ; \mathbb{R}^{L}\right)$ such that

(1) $\lim _{k \rightarrow \infty} u_{\tau_{k}}(t)=u(t)$ in $L^{1}\left(\Omega ; \mathbb{R}^{m}\right)$ for all $t \in[0, T]$ and $(u, \bar{D} u) \in L^{\infty}\left(0, T ; W^{1, \mu}\left(\Omega ; \mathbb{R}^{m}\right)\right)$;

(2) $\lim _{k \rightarrow \infty} \lambda_{\tau_{k}}(t)=\lambda(t)$ weakly in $W^{1,2}\left(\Omega ; \mathbb{R}^{L}\right)$ for all $t \in[0, T]$, and $\lambda \in B V\left(0, T ; L^{1}\left(\Omega ; \mathbb{R}^{L}\right)\right)$;

(3) $\lim _{\xi \in \Xi} \Gamma_{\tau_{k}}\left(t, q_{\tau_{\xi}}\right)=\Gamma(t, q(t))$ for all $t \in[0, T]$;

(4) $w^{*}-\lim \eta_{\tau_{\xi}}(t)=\eta(t)$ for all $t \in[0, T]$ in the sense of net convergence. Moreover, every limit $q=$ $(u, \bar{D} u, \nu, \lambda)$ is a solution according to Definition 3.3 .

Proof. We recall that the existence of a solution to (3.18) is shown in Proposition 3.5. We divide the proof into five steps. The proof follows the one of [17], Theorem 3.4.

Step 1. We first demonstrate Claim 2. By Helly's principle [18], Corollary 2.8, we get $\lambda_{\tau_{k}}(t) \rightarrow \lambda(t)$ weakly in $W^{1,2}\left(\Omega ; \mathbb{R}^{L}\right)$ for all $t \in[0, T]$ and $\lambda \in B V\left(0, T ; L^{1}\left(\Omega ; \mathbb{R}^{L}\right)\right)$. Moreover, Proposition 3.6 is applicable; (3.27) then implies that, again up to a selection of a subsequence, $\lambda_{\tau_{k}}(t) \rightarrow \lambda(t)$ weakly in $W^{1,2}\left(\Omega ; \mathbb{R}^{L}\right)$. This proves Claim 2. We recall the definition $\left.\hat{\Gamma}_{\tau}(t):=\Gamma_{\tau}\left(t, q_{\tau}(t)\right)\right)$ of the approximate Gibbs energy; we remark that (3.28) shows that $\hat{\Gamma}_{\tau_{k}}$ converges in the sense that there is a $\hat{\Gamma} \in B V(0, T)$ such that $\hat{\Gamma}_{\tau_{k}}(t) \rightarrow \hat{\Gamma}(t)$ pointwise as $k \rightarrow \infty$. Step 2. We now establish the convergence in Claim 1 and follow [17], Proof of Theorem 3.4. For a fixed $t \in[0, T]$, take a sequence $\left\{\theta\left(t, \tau_{k}\right)\right\}_{k \in \mathbb{N}}$ such that $\theta\left(t, \tau_{k}\right) \rightarrow t$ as $k \rightarrow \infty$. We write $q_{\tau_{k}}(t) \in S\left(\theta\left(t, \tau_{k}\right)\right)$. We use Claim 2 established for the subsequence of $\lambda_{\tau_{k}}(t)$ in Step 1 in conjunction with Proposition 4.1 to deduce the convergence of Claim 1. The regularity stated in Claim 1 follows from the a priori estimate (3.26) of Proposition 3.6; we can select a subsequence (not relabeled) such that $u_{\tau_{k}} \rightarrow u$ weakly* in $L^{\infty}\left(0, T ; W^{1, \mu}\left(\Omega ; \mathbb{R}^{m}\right)\right)$ as $k \rightarrow \infty$.

Step 3. Claim 4 can be proved similarly as in [17]. Notice that $\left\|\eta_{\tau}\right\|_{M\left(\bar{\Omega} ; \mathbb{R}^{m \times n}\right)}$ is bounded, which means that $\sigma_{\tau}(t)(\bar{\Omega})$ is bounded. Consequently, $\eta_{\tau}(t)\left(\bar{\Omega} \times \beta_{\mathcal{F}} \mathbb{R}^{m \times n}\right)$ is uniformly bounded and lives in a ball $B \subset$ $M\left(\bar{\Omega} \times \beta_{\mathcal{F}} \mathbb{R}^{m \times n}\right)$. If we consider $\eta_{\tau}=\left\{\eta_{\tau}(t)\right\}_{t \in[0, T]}$ as an element of $B^{[0, T]}$, which is a weakly* compact set by the Tychonoff's theorem, we find that there is a finer convergent net. Thus, there exists a limit of a subsequence of $\left\{\eta_{\tau_{\xi}}\right\}_{\xi \in \Xi}$, which we denote $\eta(t)$.

Step 4. Let us now prove Claim 3. We already know that for a fixed time $t$, the convergence $q_{\tau_{k}}(t) \rightarrow q(t)$ weakly in $W^{1, \mu}\left(\Omega ; \mathbb{R}^{m}\right) \times G D M_{\mathcal{F}}^{u_{D}}\left(\Omega ; \mathbb{R}^{m \times n}\right) \times W^{1,2}\left(\Omega ; \mathbb{R}^{L}\right)$ holds. Since $q_{\tau_{k}}(t) \in S\left(\theta\left(t, \tau_{k}\right)\right)$, it follows from Proposition 3.4 that $q(t) \in S(t) ; q(t) \in Q$ by definition of $Q$. In addition, $\lim _{k \rightarrow \infty} \Gamma_{\tau_{k}}\left(t, q_{\tau_{k}}(t)\right)=\Gamma(t, q(t))$, again by Proposition 3.4. Since $\hat{\Gamma}_{\tau}(t):=\Gamma_{\tau}\left(t, q_{\tau}(t)\right)$, the pointwise convergence $\hat{\Gamma}_{\tau_{k}}(t) \rightarrow \hat{\Gamma}(t)$ established in Step 1 in conjunction with the stability estimate (3.19) of Proposition 3.5 implies that $\Gamma(t, q(t)) \leq \hat{\Gamma}(t) \leq$ $\Gamma(t, \tilde{q})+D(q(t), \tilde{q})$. Since $q(t) \in Q$, the choice $\tilde{q}:=q(t)$ is admissible and yields $\hat{\Gamma}(t)=\Gamma(t, q(t))$. This establishes the validity of Claim 3 .

Step 5. Now we can pass to the limit in (3.20), with $t_{1}:=0$ and $t_{2}:=t$ for some $t \in[0, T]$, where it is not restrictive to suppose that $t$ is a grid point to some partition of $[0, T]$. In particular, (3.20) is valid also for all 
refinements of this partition. As in the previous argument, we use a consequence of the stability estimate (3.19) of Proposition 3.5, $\lim _{k \rightarrow \infty} \Gamma_{\tau_{k}}\left(t, q_{\tau_{k}}\right)=\Gamma(t, q(t))$. The pointwise convergence of $\lambda_{\tau_{k}}$ (Claim 2, established in the first step) in combination with Fatou's Lemma shows the sequential weak lower semicontinuity of Diss $(\cdot,[0, t])$, as defined in (3.4).

Moreover, $\lim _{k \rightarrow \infty} \int_{0}^{t} F\left(\theta, q_{\tau_{k}}(\theta)\right) \mathrm{d} \theta=\int_{0}^{t} F(\theta, q(\theta)) \mathrm{d} \theta$ due to the continuity of the forcing term. As $\left\{u_{\tau_{k}}\right\}$ has the same weak* limit as $\left\{u_{\tau_{k}}\left(\cdot-\tau_{k}\right)\right\}$, we find that both terms expressing the work of external forces in (3.20) converge to the same expression, namely $\int_{0}^{t} \frac{\partial}{\partial \theta} F(\theta, q(\theta)) \mathrm{d} \theta$. Now we pass to the limit with both sides of (3.20); to this behalf, we set

$$
e(t):=\Gamma(t, q(t))-\Gamma(0, q(0))+\operatorname{Diss}(q,[0, t])-\int_{0}^{t} \frac{\partial}{\partial \theta} F(\theta, q(\theta)) \mathrm{d} \theta .
$$

Then $e(t)=0$ at any point $t$ of the form $t=k \tau \in[0, T]$ with $1 \leq k \leq N$ by (3.20). The set of such points is dense in $[0, T]$. Therefore, (4.2) also holds for every point of continuity of $e$. All functions involved in (4.2) are functions of bounded variation; thus, they are continuous everywhere except for at most countably many points. Therefore, (4.2) holds a.e. in $[0, T]$.

We now follow the ideas of [17], Proof of Theorem 3.4, to show that $e(t)=0$ for every $t \in[0, T]$. Indeed, as $\lambda \in B V\left(0, T ; L^{1}\left(\Omega ; \mathbb{R}^{L}\right)\right)$ by Claim 2 established in Step 1, the left-sided and the right-sided limits exist for every $t \in[0, T]$. Let us consider a point $\theta \in[0, T]$ where $e$, given by (4.2), is not continuous, and denote $\lambda^{+}(\theta):=\lim _{t \backslash \theta} \lambda(t)$ and $\lambda^{-}(\theta):=\lim _{t \gamma_{\theta}} \lambda(t)$. By Proposition 4.1, there exist also the weak limits $u^{+}(\theta):=$ $\lim _{t \searrow \theta} u(t)$ and $u^{-}(\theta):=\lim _{t \nearrow \theta} u(t)$. Therefore $q^{+}(\theta):=\lim _{t \searrow \theta} q(t)$ and $q^{-}(\theta):=\lim _{t \nearrow \theta} q(t)$ exist in the weak topology. Let us also define $\Gamma^{+}(t):=\lim _{t \backslash \theta} \Gamma(t, q(t))$ and $\Gamma^{-}(t)(\theta):=\lim _{t} \nearrow_{\theta} \Gamma(t, q(t))$. As shown in Step 4, $\Gamma^{+}(\theta)=\Gamma\left(\theta, q^{+}(\theta)\right)$ and $\Gamma^{-}(\theta)=\Gamma\left(\theta, q^{-}(\theta)\right)$. We test the stability of $q(\theta) \in S(\theta)$ (established in Step 4) by $\tilde{q}:=q^{+}(\theta)$ and obtain

$$
\Gamma(\theta, q(\theta)) \leq \Gamma\left(\theta, q^{+}(\theta)\right)+D\left(q(\theta), q^{+}(\theta)\right)=\Gamma^{+}(\theta)+D\left(q(\theta), q^{+}(\theta)\right)
$$

Similarly, $q^{-}(\theta)$ is stable as a limit of stable configurations by Proposition 3.4. Hence, with (4.3),

$$
\begin{aligned}
\Gamma^{-}(\theta) & =\Gamma\left(\theta, q^{-}(\theta)\right) \leq \Gamma(\theta, q(\theta))+D\left(q^{-}(\theta), q(\theta)\right) \\
& \leq \Gamma^{+}(\theta)+D\left(q(\theta), q^{+}(\theta)\right)+D\left(q^{-}(\theta), q(\theta)\right) .
\end{aligned}
$$

By definition (1.15), Diss $(q,[s, t])=\operatorname{Diss}(q,[s, \theta])+\operatorname{Diss}(q,[\theta, t])$ for $s<\theta<t$. Further, $\lim _{t}{ }_{\theta} \operatorname{Diss}(q,[t, \theta])=$ $D\left(q^{-}(\theta), q(\theta)\right)$ and $\lim _{t \backslash \theta} \operatorname{Diss}(q,[\theta, t])=D\left(q(\theta), q^{+}(\theta)\right)$. Passing to the limit in (4.2), we find

$$
\Gamma^{+}(\theta)-\Gamma^{-}(\theta)+D\left(q^{-}(\theta), q(\theta)\right)+D\left(q(\theta), q^{+}(\theta)\right)=0
$$

Thus, (4.4) is in fact an equality (and thus the same holds for (4.3)). Thus for $\theta>0$ one gets with (4.4)

$$
e(\theta)-\lim _{t \nearrow \theta} e(t)=\Gamma(\theta, q(\theta))-\Gamma^{-}(\theta)+D\left(q^{-}(\theta), q(\theta)\right)=0 .
$$

This shows continuity of $e$ from the left. Similarly, we can show its continuity from the right. Hence, we have $e(t)=0$ for all $t \in[0, T]$. The energy inequality (3.12) is equivalent to $e\left(t_{1}\right)=e\left(t_{2}\right)$, which has just been shown.

\section{Appendix A. Compactifications and DiPerna-Majda measures}

The construction of DiPerna-Majda measures introduces a compactification of the target space $\mathbb{R}^{d}$. A compactification of $\mathbb{R}^{d}$ is a compact set, denoted by $\beta_{\mathcal{F}} \mathbb{R}^{d}$, into which $\mathbb{R}^{d}$ is densely and homeomorphically embedded. 
Various compactifications of $\mathbb{R}^{d}$ exist, the two extreme cases being the (Alexandroff) one-point compactification, where a point at infinity is added, and the Stone-Čech compactification $\beta \mathbb{R}^{d}$.

In a nutshell, compactifications of a locally compact Hausdorff space $X$ can be constructed as follows. Let $B C(X)$ denote the bounded continuous functions $f: X \rightarrow \mathbb{R}$. Consider a subset $\mathcal{F} \subset B C(X)$ and let $J$ be an index set for $\mathcal{F}$. We can identify each point $x$ in $X$ with its evaluation $h(x):=\left(f_{j}(x)\right)_{j \in J}$, and the latter is compact by Tychonoff's theorem. Furthermore, suppose that $\mathcal{F}$ separates points from closed sets; that is, for a closed set $C \subset X$ and a point $x \notin C$, there exists $f \in \mathcal{F}$ such that $f(x) \notin \overline{f(C)}$. Then every $f$ in the smallest closed algebra in $B C(X)$ containing $\mathcal{F}$ has a continuous extension to the compactification $\beta_{\mathcal{F}} X$ of $X$ by $\mathcal{F}$ [11], Proposition 4.56. Here, closeness is understood in the maximum norm. Since in this situation, the space of continuous bounded functions on $X$ is thus isometrically isomorphic to the space of continuous functions on $\beta_{\mathcal{F}} X$, we shall not distinguish between elements of $\mathcal{F}$ and their unique continuous extensions on $\beta_{\mathcal{F}} X$. Likewise, we silently identify $X$ with its image in $\beta_{\mathcal{F}} X$.

$\mathcal{F}_{1}=B C(X)$ yields the Stone-Čech compactification, while $\mathcal{F}_{0}=C_{0}(X)$ gives the one-point compactification. We choose an intermediate compactification.

An algebra is completely regular if it is closed with respect to the maximum norm, contains the constants and separates points from closed subsets. It is known [8], Section 3.12.21, that there is a one-to-one correspondence $\mathcal{F} \mapsto \beta_{\mathcal{F}} \mathbb{R}^{d}$ between complete, separable subalgebras of $B C\left(\mathbb{R}^{d}\right)$ and metrisable compactifications of $\mathbb{R}^{d}$. (In particular, the Stone-Čech compactification $\beta X$ is not metrisable unless $X$ was already compact.) It is easy to see that $\beta_{\mathcal{F}_{1}} \mathbb{R}^{d} \subset \beta_{\mathcal{F}_{2}} \mathbb{R}^{d}$ if $\mathcal{F}_{1} \subset \mathcal{F}_{2}$. We choose $\mathcal{F} \subset B C\left(\mathbb{R}^{d}\right)$ to be the set of functions $f$ which have radial limits $\lim _{r \rightarrow \infty} f(r s)$ for every direction $s$. Obviously $\mathcal{F} \supset C_{0}\left(\mathbb{R}^{d}\right)$, and thus $\mathcal{F}$ separates points from compact sets by Urysohn's Lemma.

Acknowledgements. This work was initiated when M.K. visited the Department of Mathematical Sciences at the University of Bath. The hospitality and support of the department is gratefully acknowledged. J.Z. thanks the Nečas Centre for supporting a one-month research stay in Prague in November 2006. M.K. was supported by the grants 1 M06031 (MŠMT ČR), VZ6840770021 (MŠMT ČR), and by the EU project MRTN-CT-2004-505226 "MULTIMAT". J.Z. gratefully acknowledges the financial support of the EPSRC through an Advanced Research Fellowship (GR/S99037 / 1).

\section{REFERENCES}

[1] L. Ambrosio, N. Fusco and D. Pallara, Functions of bounded variation and free discontinuity problems, Oxford Mathematical Monographs. The Clarendon Press Oxford University Press, New York (2000).

[2] J.M. Ball, A version of the fundamental theorem for Young measures, in PDEs and continuum models of phase transitions (Nice, 1988), M. Rascle, D. Serre and M. Slemrod Eds., Springer, Berlin (1989) 207-215.

[3] J.M. Ball and R.D. James, Fine phase mixtures as minimizers of energy. Arch. Ration. Mech. Anal. 100 (1987) 13-52.

[4] S. Conti and M. Ortiz, Dislocation microstructures and the effective behavior of single crystals. Arch. Ration. Mech. Anal. 176 (2005) 103-147.

[5] G. Dal Maso, A. DeSimone, M.G. Mora and M. Morini, A vanishing viscosity approach to quasistatic evolution in plasticity with softening. Technical report, Scuola Normale Superiore, Pisa (2006).

[6] G. Dal Maso, A. DeSimone, M.G. Mora and M. Morini, Time-dependent systems of generalized Young measures. Netw. Heterog. Media 2 (2007) 1-36 (electronic).

[7] R.J. DiPerna and A.J. Majda, Oscillations and concentrations in weak solutions of the incompressible fluid equations. Comm. Math. Phys. 108 (1987) 667-689.

[8] R. Engelking, General topology. Translated from the Polish by the author, Monografie Matematyczne 60 [Mathematical Monographs]. PWN - Polish Scientific Publishers, Warsaw (1977).

[9] L.C. Evans, Partial differential equations, Graduate Studies in Mathematics 19. American Mathematical Society, Providence, USA (1998).

[10] L.C. Evans and R.F. Gariepy, Measure theory and fine properties of functions, Studies in Advanced Mathematics. CRC Press, Boca Raton, USA (1992).

[11] G.B. Folland, Real Analysis: Modern Techniques and Their Applications, Pure and Applied Mathematics. John Wiley \& Sons Inc., New York, first edition (1999); Wiley-Interscience, second edition.

[12] G. Francfort and A. Mielke, Existence results for a class of rate-independent material models with nonconvex elastic energies. J. Reine Angew. Math. 595 (2006) 55-91. 
[13] A. Kałamajska and M. Kružík, Oscillations and concentrations in sequences of gradients. ESAIM: COCV 14 (2008) 71-104.

[14] M. Kružík and T. Roubíček, On the measures of DiPerna and Majda. Math. Bohem. 122 (1997) 383-399.

[15] A. Mainik and A. Mielke, Existence results for energetic models for rate-independent systems. Calc. Var. Partial Differential Equations 22 (2005) 73-99.

[16] A. Mielke, Evolution of rate-independent systems, in Evolutionary equations II, Handb. Differ. Equ., Elsevier/North-Holland, Amsterdam (2005) 461-559.

[17] A. Mielke and T. Roubíček, A rate-independent model for inelastic behavior of shape-memory alloys. Multiscale Model. Simul. 1 (2003) 571-597 (electronic).

[18] A. Mielke, F. Theil and V.I. Levitas, A variational formulation of rate-independent phase transformations using an extremum principle. Arch. Ration. Mech. Anal. 162 (2002) 137-177.

[19] M. Ortiz and E.A. Repetto, Nonconvex energy minimization and dislocation structures in ductile single crystals. J. Mech. Phys. Solids 47 (1999) 397-462.

[20] T. Roubíček, Relaxation in optimization theory and variational calculus, de Gruyter Series in Nonlinear Analysis and Applications 4. Walter de Gruyter \& Co., Berlin (1997).

[21] M.E. Schonbek, Convergence of solutions to nonlinear dispersive equations. Comm. Partial Differential Equations 7 (1982) 959-1000.

[22] J. Souček, Spaces of functions on domain $\Omega$, whose $k$-th derivatives are measures defined on $\bar{\Omega}$. Časopis Pěst. Mat. 97 (1972) $10-46$.

[23] L. Tartar, Compensated compactness and applications to partial differential equations, in Nonlinear analysis and mechanics: Heriot-Watt Symposium IV, Pitman, Boston, USA (1979) 136-212. 